\title{
Localized Emitting State and Energy Transfer Properties of Quadrupolar Chromophores and (Multi)Branched Derivatives
}

\author{
Linyin Yan, Xudong Chen, Qingguo He, Yingying Wang, Xuefei Wang, Qianjin Guo,* Fenglian Bai, \\ and Andong Xia*
}

The State Key Laboratory of Molecular Reaction Dynamics, Institute of Chemistry, Chinese Academy of Sciences, Beijing 100190, P. R. China

Beijing National Laboratory for Molecular Sciences (BNLMS), Institute of Chemistry, Chinese Academy of Sciences, Beijing 100190, P. R. China

\section{Damir Aumiler and Silvije Vdović}

The State Key Laboratory of Molecular Reaction Dynamics, Institute of Chemistry, Chinese Academy of Sciences, Beijing 100190, P. R. China

Institute of Physics, Bijenička cesta 46, 10000 Zagreb, Croatia

\section{ShengHien Lin*}

Department of Applied Chemistry, National Chiao Tung University, Hsinchu, Taiwan 30010, China

\section{Supporting Information}

\begin{abstract}
In order to better understand the nature of intramolecular charge and energy transfer in multibranched molecules, we have synthesized and studied the photophysical properties of a monomer quadrupolar chromophore with donor-acceptor-donor (D-A-D) electronic push-pull structure, together with its V-shaped dimer and star-shaped trimers. The comparison of steady-state absorption spectra and fluorescence excitation anisotropy spectra of these chromophores show evidence of weak interaction (such as charge and energy transfer) among the branches. Moreover, similar fluorescence and solvation behavior of monomer and branched chromophores (dimer and trimer) implies that the interaction

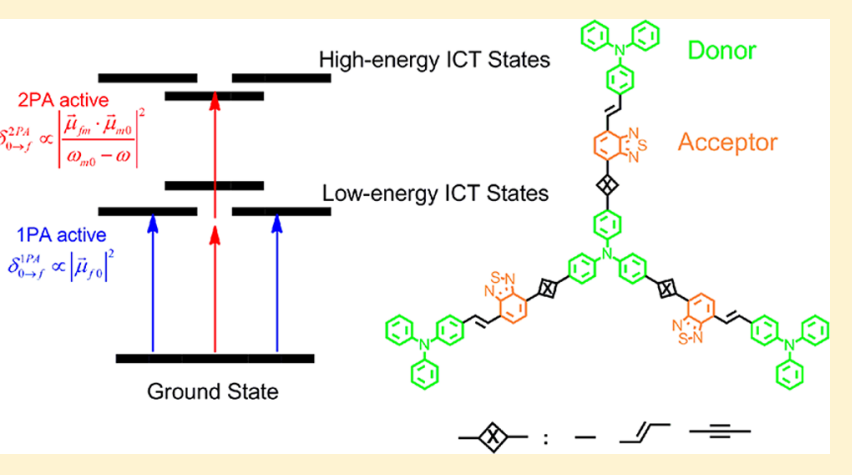
among the branches is not strong enough to make a significant distinction between these molecules, due to the weak interaction and intrinsic structural disorder in branched molecules. Furthermore, the interaction between the branches can be enhanced by inserting $\pi$ bridge spacers $(-\mathrm{C}=\mathrm{C}-$ or $-\mathrm{C} \equiv \mathrm{C}-$ ) between the core donor and the acceptor. This improvement leads to a remarkable enhancement of two-photon cross-sections, indicating that the interbranch interaction results in the amplification of transition dipole moments between ground states and excited states. The interpretations of the observed photophysical properties are further supported by theoretical investigation, which reveal that the changes of the transition dipole moments of the branched quadrupolar chromophores play a critical role in observed the two-photon absorption (2PA) cross-section for an intramolecular charge transfer (ICT) state interaction in the multibranched quadrupolar chromophores.
\end{abstract}

\section{INTRODUCTION}

Recent advances in the synthesis of novel organic materials with intramolecular charge transfer (ICT) properties resulted in a broad area of applications ranging from new optoelectronic devices such as electroluminescence devices, ${ }^{1-3}$ thin film transistors, and solar cells, ${ }^{4,5}$ to the nonlinear optical materials. $^{6-9}$ The linear ICT compounds commonly composed of conjugated electron-donating (D) and electron-accepting (A) groups connected through a conjugation $\pi$-linker have already been widely reported, and their photophysical properties and applications have been extensively studied. ${ }^{10-17}$ In the quest of improving photophysical properties, multibranched molecules with two or more linear ICT moieties were synthesized. ${ }^{9,18-20}$

Received: June 2, 2012

Revised: July 13, 2012

Published: August 10, 2012 


\section{Scheme 1. Chemical Structures of Chromophores 1-5}

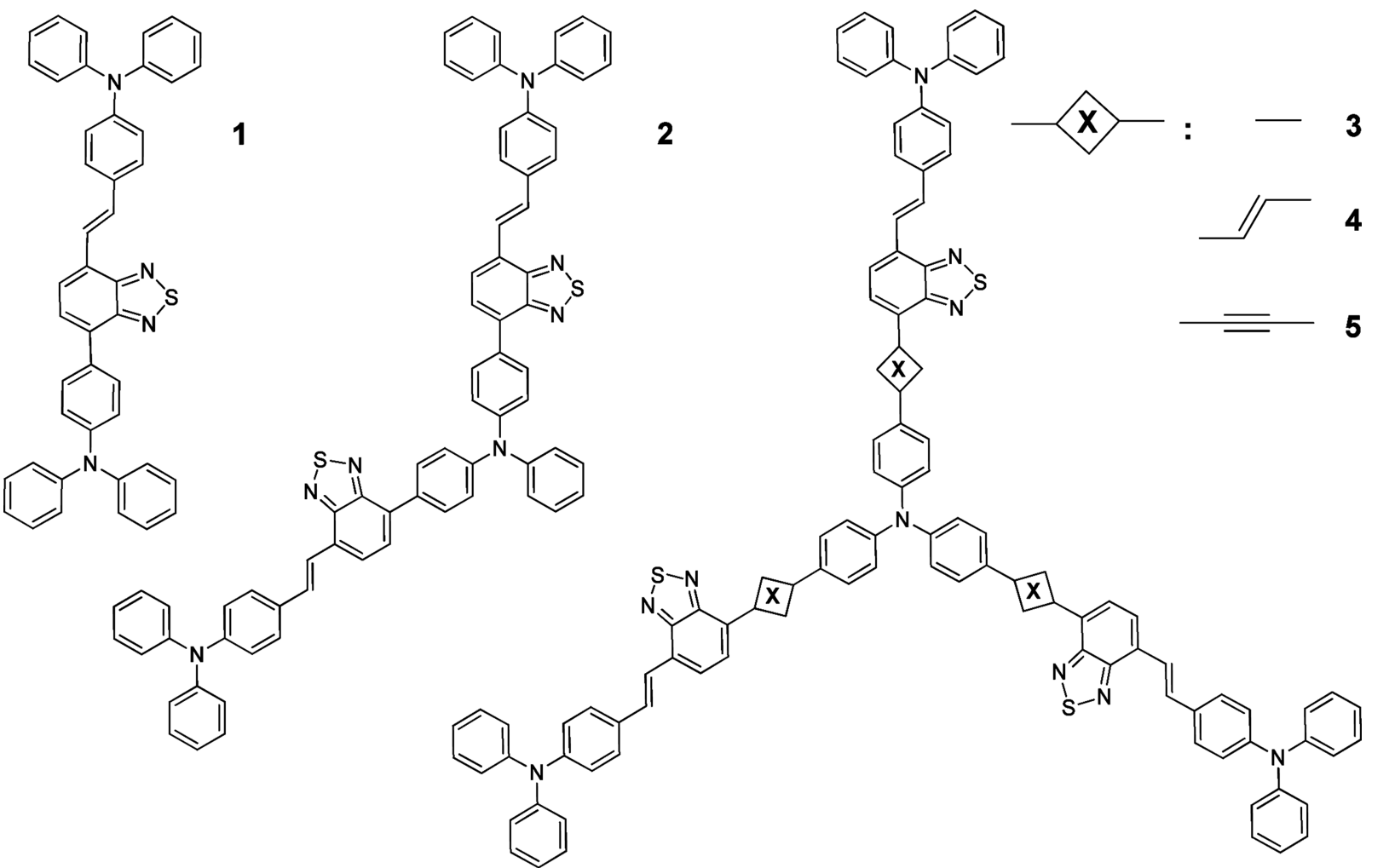

In general, it is expected that the delocalization could be extended by connecting two or more ICT moieties through conjugation bridges, for example, triphenyleamine group. In this way, some special photophysical properties could be obtained. However, unlike the linear conjugation systems with photophysical properties strongly dependent on the conjugation extension, ${ }^{21-24}$ previous literature and our results show that the increase of branch number does not guarantee an essential change of the steady-state spectra. For instance, steady-state spectral methods and quantum chemistry calculations have widely been applied to study the ICT behavior of a group of triple-branched structures, and the results showed that the emitting state is mainly localized on a single branch because of the structural disorder in branched ICT molecules. ${ }^{25-27}$ In that case, significant cooperative enhancement of the twophoton cross-section of triple-branched structures could not be observed, in comparison with their counterpart linear moiety. ${ }^{9,19,20,25}$ However, considering the short spatial distance between the branches and the identical transition moment of these moieties, it is still not likely that the branches are fully independent of each other. Energy transfer and interactions among the branches in the excited state should still exist within these compounds. ${ }^{18,26-30}$ Obviously, these crucial spectral properties are closely related to the chemical structure of the molecules. The nature of optical excitations and the degree of ICT in multiple branched push-pull molecules with many complex structural architectures remains not well understood because of the intrinsic complexity of the ICT chromophores, which are strongly affected by geometric confinement and charge symmetry, as well as their surrounding environment.

In order to obtain an overall understanding of these interbranch interactions and their impact on the molecular photophysical properties, in this article, we have conducted both experimental and computational investigations on a series of newly synthesized molecules (chromophores 1-5, molecular structures shown in Scheme 1). These branched ICT compounds are formed by the gathering of monomer quadrupolar $(\mathrm{D}-\pi-\mathrm{A}-\pi-\mathrm{D})$ units in which triphenylamine was used as the branching and donor units (D), benzothiadiazole as the acceptor units (A), and single-, double- or triplebonds as the $\pi$-bridge $(\pi)$ (Scheme 1$)$. In this article, the effect of branching of these quadrupolar chromophores on both onephoton and two-photon absorption properties have been studied by combining various experimental and theoretical approaches. The experimental results show that the excited ICT states of branched quadrupolar chromophores exhibited a localized feature on one of the branches, where the degree of ICT and polar nature of the excited ICT state is almost identical to that of quadrupolar monomer. Moreover, in addition to the basic TD-DFT calculations, Frenkel exciton model was further used to interpret the complicated spectral properties of the coupled ICT states in these branched quadrupolar chromophores.

\section{MATERIALS AND METHODS}

2.1. Materials. The synthesis of all compounds have been reported elsewhere previously. ${ }^{31,32}$ All solvents used in our work including chloroform, cyclohexane, dichloromethane, acetone, acetonitrile, toluene, ethyl alcohol, and methyl alcohol were analytical grade and purchased from the Beijing Chemical Plant.

2.2. Absorption and Fluorescence Measurements. Absorption spectra were detected by a UV-vis spectrophotometer (U-3010, Shimadzu). Fluorescence spectra were measured with a fluorescence spectrophotometer (F4600, Hitachi). All experiments were performed at room temperature. Fluorescence excitation anisotropy spectra were measured by a 
Scheme 2. Schematic Electronic Level Diagrams within the Excitonic Model for (a) a Monomer Quadrupole (D-A-D), (b) a V-Shaped Dimer Comprising Two Quadrupolar Branches $\left(\mathrm{D}(-\mathrm{A}-\mathrm{D})_{2}, C_{2}\right.$ Symmetry), and (C) a Branched Structure Obtained by Grafting Three Quadrupoles $\left(\mathrm{D}(-\mathrm{A}-\mathrm{D})_{3}, C_{3 h} \text { Symmetry }\right)^{a}$

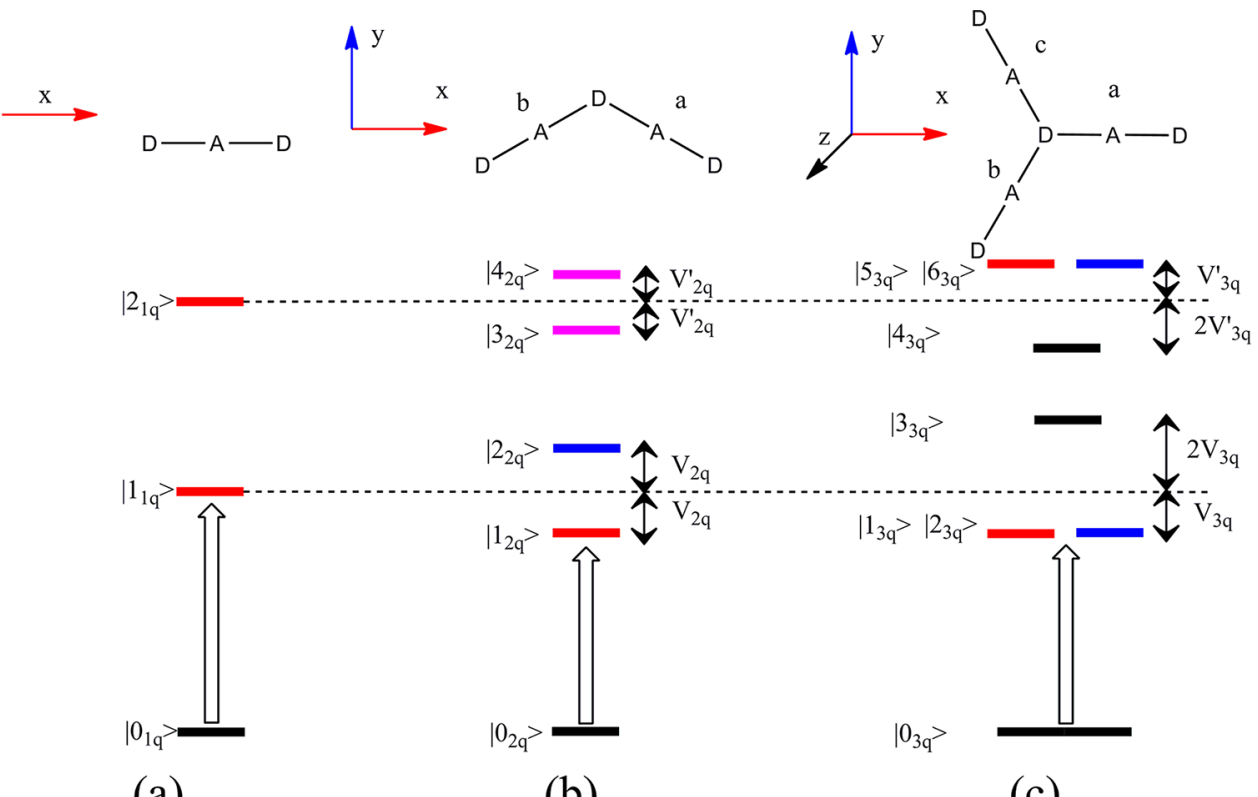

(a)

(b)

(c)

$\left.a_{\mid 0_{m q}}\right\rangle$ denotes the ground state, and $\left|n_{m q}\right\rangle(n=1,2, \ldots, 6)$ represents the $n$th excited state, where $m=1,2,3$ is used to make a distinction among different structures with 1,2 , and 3 branches. $V\left(V_{2 q}, V_{2 q}^{\prime}, V_{3 q}\right.$ and $\left.V^{\prime}{ }_{3 q}\right)$ denotes the interbranch coupling for the lowest excited state, and the subscript $\mathrm{q}$ represents quadrupolar chromophore. The coordinate $(x, y$, and $z)$ is also shown for recognizing the transition dipole moment direction.

fluorescence spectrophotometer (F4600, HITACHI) with two polarizers in excitation and detection light routes, respectively. The anisotropy $(r)$ was calculated with

$$
r=\frac{I_{\|}-G I_{\perp}}{I_{\|}+2 G I_{\perp}}
$$

where $I_{\|}$and $I_{\perp}$ are the polarized fluorescence intensities parallel and perpendicular with excitation polarization, respectively; $G\left(G=I_{\perp} / I_{\|}\right)$is the geometrical factor of fluorescence spectrophotometer when the excitation is vertically polarized. ${ }^{33,34}$ To avoid fast rotation of a molecule in solution, samples were dissolved in toluene solutions with saturated polystyrene, and fluorescence excitation anisotropy spectra were measured in an isotropic polystyrene film during measurement. The fluorescence lifetimes of all the compounds were measured with the excitation at $460 \mathrm{~nm}$ and emission at $600 \mathrm{~nm}$ by means of a time-correlated single photon counting (TCSPC) apparatus with an instrument response function (IRF) of about 200 ps. The concentration of the samples for fluorescence quantum yield and fluorescence decay measurements was maintained to keep the optical density below 0.1 at the excitation wavelength in a $10 \mathrm{~mm}$ cuvette in order to minimize the self-absorption effect.

2.3. Two-Photon Excitation Measurements. Twophoton excited fluorescence (TPEF) were performed using a femtosecond pulsed laser (120 fs, $76 \mathrm{MHz}, 710-950 \mathrm{~nm})$ from a Ti:Sapphire laser (Mira-900F, Coherent, USA) pumped by a continuous wave $532 \mathrm{~nm}$ laser (Verdi-5, Coherent, USA). The TPEF was collected with a $90^{\circ}$ geometry and recorded with a liquid nitrogen cooled CCD detector (Jobin Yvon, CCD-3000 $\mathrm{V})$ attached to a spectrophotometer (Jobin Yvon TRIAX 320). The two-photon absorption (2PA) cross-sections $(\delta)$ of samples were obtained following the method described in refs 33 and $35-37$ by the use of the eq 2 :

$$
\delta_{\mathrm{s}}=\frac{S_{\mathrm{s}} \eta_{\mathrm{r}} \Phi_{\mathrm{r}} C_{\mathrm{r}}}{S_{\mathrm{r}} \eta_{\mathrm{s}} \Phi_{\mathrm{s}} C_{\mathrm{s}}} \delta_{\mathrm{r}}
$$

where $\Phi$ and $S$, represent the collection efficiency of the optical system and integrated intensity of the TPEF, respectively. C stands for the sample concentration and $\eta$ for the fluorescence quantum yield. Subscripts s and $\mathrm{r}$ refer to sample and reference, respectively. Rhodamine B in methanol was used as reference, ${ }^{35}$ and the appropriate solvent-related refractive index correction for the quantum yield was also made. Sample solutions $1 \times$ $10^{-4} \mathrm{M}$ in toluene for all compounds were used in two photon experiments. The TPA spectra were measured every $10 \mathrm{~nm}$ stepped from 710 to $950 \mathrm{~nm}$. The laser power was kept the same at different wavelengths during the TPEF measurements, where the quadratic dependence of the fluorescence intensity on the excitation intensity was verified for each data point. The sample solution was kept in a flow cell ( $10 \mathrm{~mm}$ path length) in which saturation or photodegradation did not occur. Considering the different pulse widths ranging from 120 to 150 fs within $710-950 \mathrm{~nm}$ and the possible concentration uncertainty during sample solution preparation, the experimental uncertainty did not exceed $\pm 10 \%$.

\section{THEORETICAL METHODS}

3.1. Frenkel Exciton Model. In this work, we use the Frenkel exciton model to analyze the transition dipole moments of multibranched quadrupolar chromophores 2-5 together with their single-branched counterpart chromophore $1^{38-41}$ This model has been successfully applied to multibranched chromophores systems, ${ }^{25,26,42-44}$ which assumes an electrostatic interaction between monomers, and the inter- 
action is small compared to the typical energy gaps between electronic states. The energy level splittings of the excitonic states caused by the coupling between the branches are shown in Scheme 2.

In the Frenkel exciton model, the direction of molecular transition dipole moment depends on the interbranch coupling and the charge delocalization. ${ }^{35}$ For the single quadrupole molecule of chromophore 1 with linear quadrupolar geometry, the transition dipole moments of the first and second excited states $\left(\left|1_{1 q}\right\rangle\right.$ and $\left.\left|2_{1 q}\right\rangle\right)$ are both in the direction of the long axis of the molecule (shown in Scheme 2). This situation changes when another branch is attached on the donor group to generate a $\mathrm{V}$-shaped chromophore 2 . The original excited states $\left|1_{1 q}\right\rangle$ and $\left|2_{1 q}\right\rangle$ split into two new excited states, $\left|1_{2 q}\right\rangle,\left|2_{2 q}\right\rangle$ and I $\left.3_{2 q}\right\rangle,\left|4_{2 q}\right\rangle$, respectively. The transition dipole moments also changed in both amplitude and direction. Given the coordinate axes as shown in Scheme 2, if the ground state is neutral, the transition dipole moment from ground state to state $\left|1_{2 q}\right\rangle$ is along the $x$ axis, while the transition dipole moment from ground state to state $\left|2_{2 \mathrm{q}}\right\rangle$ is along the $y$ axis. Meanwhile, though the directions of transition dipole moments from ground state to states $\left|3_{2 q}\right\rangle$ and $\left|4_{2 q}\right\rangle$ are different from each other, they both contain components from both $x$ and $y$ directions. In the case of three-branched molecules with $C_{3 h}$ symmetry, for example, chromophores 3,4 , and 5 , group theory and the Gram-Schmidt orthogonalization procedure can be used to describe the excited states shown in Table S2 (Supporting Information) and the coupling among different branches. ${ }^{45}$ With the $C_{3 h}$ symmetry, from the interaction between three single-quadrupolar branches, the lowest excited state $\left(\left|1_{1 \mathrm{q}}\right\rangle\right.$ of the single branch is then split into three states, where two degenerated lower excited states $\left(\left|1_{3 \mathrm{q}}\right\rangle=\left(2\left|1_{\mathrm{q}(\mathrm{a})}\right\rangle-\right.\right.$ $\left.\left|1_{\mathrm{q}(\mathrm{b})}\right\rangle-\left|1_{\mathrm{q}(\mathrm{c})}\right\rangle\right) / \sqrt{ } 6$ and $\left.\left|2_{3 \mathrm{q}}\right\rangle=\left(\left|1_{\mathrm{q}(\mathrm{b})}\right\rangle-\left|1_{\mathrm{q}(\mathrm{c})}\right\rangle\right) / \sqrt{ } 2\right)$ and one higher excited state $\left(\left|3_{3 q}\right\rangle=\left(\left|1_{q(a)}\right\rangle+\left|1_{q(b)}\right\rangle+\left|1_{q(c)}\right\rangle\right) / \sqrt{ } 3\right)$ are obtained. The state $\left|3_{3 q}\right\rangle$ has the same $C_{3 h}$ symmetry as the ground state. When the ground state is neutral, the orientation of transition dipole moment from ground state to $\left|1_{3 \mathrm{q}}\right\rangle$ and $\mid$ $\left.2_{3 \mathrm{q}}\right\rangle$ are along the $x$ and $y$ axes, respectively, while the transition dipole moment from ground state to the state $\left|3_{3 q}\right\rangle$ is zero. However, the thermal motion and structural disorder could break the $C_{3 h}$ symmetry of these chromophores, resulting in small transition probability from ground state to the state $\left|3_{3 q}\right\rangle$. Similarly, the higher excited state $\left|2_{1 \mathrm{q}}\right\rangle$ in the single branch is also split into three new states: $\left(\left|4_{3 q}\right\rangle,\left|5_{3 q}\right\rangle\right.$, and $\left.\left|6_{3 q}\right\rangle\right)$ with the interaction between three single-quadrupolar branches as shown in Scheme 2. Interestingly, the energy of two degenerated states $\left(\left|5_{3 q}\right\rangle\right.$ and $\left.\left|6_{3 q}\right\rangle\right)$ is higher than that of nondegenerate state $\left|4_{3 q}\right\rangle$.

3.2. Calculation of Two-Photon Absorption. In the single-beamlinearly polarized one color (the angular frequency of $\omega)$ 2PA process, the time-dependent wave equation is

$$
\hat{H} \Psi=i \hbar(\partial \Psi / \partial t)
$$

where the Hamiltonian operator can be written as

$$
\hat{H}=\hat{H}_{0}-\boldsymbol{\mu} \cdot \mathbf{E}_{0} \cos \omega t=\hat{H}_{0}-e\left|\mathbf{E}_{0}\right|(\mathbf{R} \cdot \lambda) \cos \omega t
$$

where $\boldsymbol{\mu}$ is the electronic transition dipole moment, $e$ is the elementary charge, and $\mathbf{R}$ is the corresponding spatial vector, $\lambda$ is the polarization vector of the radiation, and $\mathbf{E}_{0}$ is the amplitude vectors of incident sinusoidal electric fields.
According to the perturbation method, the second order probability and the rate of the transition from the initial state $i$ to the final state $f$ in the $2 \mathrm{PA}$ process are given by ${ }^{46}$

$$
\begin{aligned}
C_{f}^{(2)}(\omega)= & \sum_{m}\left(\frac{\boldsymbol{\mu}_{f m} \cdot \mathbf{E}_{0}}{2 \hbar}\right)\left(\frac{\boldsymbol{\mu}_{m i} \cdot \mathbf{E}_{0}}{2 \hbar}\right) \\
& \times \frac{\mathrm{e}^{\mathrm{i}\left(\omega_{f i}-2 \omega\right) t}-1}{\left(\omega_{m i}-\omega\right)\left(\omega_{f i}-2 \omega\right)}
\end{aligned}
$$

and

$$
\begin{aligned}
W_{i \rightarrow f}^{(2)}(\omega) & =\frac{\mathrm{d}}{\mathrm{d} t}\left|C_{f}^{(2)}(\omega)\right|^{2} \\
& =\frac{\pi}{8 \hbar^{4}}\left|\sum_{m} \frac{\left(\boldsymbol{\mu}_{f m} \cdot \mathbf{E}_{0}\right)\left(\boldsymbol{\mu}_{m i} \cdot \mathbf{E}_{0}\right)}{\omega_{m i}-\omega}\right|^{2} \times \delta\left(\omega_{f i}-2 \omega\right)
\end{aligned}
$$

On the basis of the Born-Oppenheimer (BO) approximation, the expression of the molecular wave function and the transition moment can be written as

$$
\begin{aligned}
& \Psi_{i v}=\Phi_{i} \Theta_{i v} \\
& \left|\boldsymbol{\mu}_{f v^{\prime} i v}\right|=\left\langle\Psi_{f v^{\prime}}, \boldsymbol{\mu} \mid \Psi_{i v}\right\rangle=\left\langle\Theta_{f v^{\prime}}\left|\left\langle\Phi_{f}|\boldsymbol{\mu}| \Phi_{i}\right\rangle\right| \Theta_{i v}\right\rangle=\left\langle\Theta_{f v^{\prime}}\left|\boldsymbol{\mu}_{f i}\right| \Theta_{i v}\right\rangle
\end{aligned}
$$

where $v$ is the vibrational state of electronic state $i$ and $v^{\prime}$ is that of $f$.

When the vibrational states $v, v^{\prime}$, and $v^{\prime \prime}$ are taken into account, the expression of the transition rate can be rewritten as

$$
\begin{aligned}
W_{i \rightarrow f}^{(2)}(\omega)= & \frac{\pi}{8 \hbar^{4}} \sum_{v} \sum_{v^{\prime}} P_{i v}\left|M_{f v^{\prime}, m v^{\prime \prime}, i v}(\omega)\right|^{2} \\
& \times D\left(\omega_{f v^{\prime}, i v}-2 \omega, \gamma_{f v^{\prime}, i v}\right)
\end{aligned}
$$

where

$$
\begin{aligned}
& M_{f v^{\prime}, m v^{\prime \prime}, i v}(\omega) \\
& \quad=\sum_{v^{\prime \prime}} \sum_{m} \frac{\left(\left\langle\Theta_{f v^{\prime}}, \boldsymbol{\mu}_{f m} \mid \Theta_{m v^{\prime \prime}}\right\rangle \cdot \mathbf{E}_{0}\right)\left(\left\langle\Theta_{m v^{\prime \prime}}\left|\boldsymbol{\mu}_{m i}\right| \Theta_{i v}\right\rangle \cdot \mathbf{E}_{0}\right)}{\omega_{m v^{\prime \prime}, i v}-\omega}
\end{aligned}
$$

and $P_{i v}$ is the Boltzmann distribution factor, and $D\left(\omega_{f v^{\prime}, i v}-\right.$ $\left.2 \omega, \gamma_{f v^{\prime}, i v}\right)$ represents the Lorentzian-shape function.

When we neglect the effect of the small vibrational displacement on transition dipole moments and exciting energy,

$$
\begin{aligned}
& \boldsymbol{\mu}_{f i} \cong \boldsymbol{\mu}_{f i}(0) \\
& \omega_{m v^{\prime \prime}, i v} \cong \omega_{m i}
\end{aligned}
$$

we obtain

$$
\boldsymbol{\mu}_{f v^{\prime} i v}=\left\langle\Theta_{f v^{\prime}}\left|\boldsymbol{\mu}_{f i}\right| \Theta_{i v}\right\rangle \cong\left\langle\Theta_{f v^{\prime}}\left|\boldsymbol{\mu}_{f i}(0)\right| \Theta_{i v}\right\rangle=\boldsymbol{\mu}_{f i}(0)\left\langle\Theta_{f v^{\prime}}, \Theta_{i v}\right\rangle
$$

and 


$$
\begin{aligned}
& M_{f v^{\prime}, m v^{\prime \prime}, i v}(\omega) \\
& =\sum_{v^{\prime \prime}} \sum_{m} \frac{\left(\boldsymbol{\mu}_{f m}(0)\left\langle\Theta_{f v^{\prime}}, \Theta_{m v^{\prime \prime}}\right\rangle \cdot \mathbf{E}_{0}\right)\left(\boldsymbol{\mu}_{m i}(0)\left\langle\Theta_{m v^{\prime \prime}} \mid \Theta_{i v}\right\rangle \cdot \mathbf{E}_{0}\right)}{\omega_{m i}-\omega} \\
& =\sum_{v^{\prime \prime}} \sum_{m} \frac{\left(\boldsymbol{\mu}_{f m}(0) \cdot \mathbf{E}_{0}\right)\left(\boldsymbol{\mu}_{m i}(0) \cdot \mathbf{E}_{0}\right)\left\langle\Theta_{f v^{\prime}} \Theta_{m v^{\prime \prime}}\right\rangle\left\langle\Theta_{m v^{\prime \prime}} \Theta_{i v}\right\rangle}{\omega_{m i}-\omega} \\
& =\left\langle\Theta_{f v^{\prime}} \Theta_{i v}\right\rangle \sum_{m} \frac{\left(\boldsymbol{\mu}_{f m}(0) \cdot \mathbf{E}_{0}\right)\left(\boldsymbol{\mu}_{m i}(0) \cdot \mathbf{E}_{0}\right)}{\omega_{m i}-\omega}
\end{aligned}
$$

So,

$$
\begin{aligned}
W_{i \rightarrow f}^{(2)}(\omega)= & \frac{\pi}{8 \hbar^{4}} \sum_{v} \sum_{v^{\prime}} P_{i v}\left|\sum_{m} \frac{\left(\boldsymbol{\mu}_{f m}(0) \cdot \mathbf{E}_{0}\right)\left(\boldsymbol{\mu}_{m i}(0) \cdot \mathbf{E}_{0}\right)}{\omega_{m i}-\omega}\right|^{2} \\
& \times\left|\left\langle\Theta_{f v^{\prime}} \mid \Theta_{i v}\right\rangle\right|^{2} \delta\left(\omega_{f v^{\prime}, i v}-2 \omega, \gamma_{f v^{\prime}, i v}\right)
\end{aligned}
$$

Hereafter, we replace the delta function $\left(\delta\left(\omega_{f v^{\prime}, i v}-\right.\right.$ $\left.\left.2 \omega, \gamma_{f v^{\prime}, i v}\right)\right)$ by the Lorentzian function $\left(D\left(\omega_{f v^{\prime}, i v}-2 \omega, \gamma_{f v^{\prime}, i v}\right)\right)$; to simplifying, we shall also replace $\boldsymbol{\mu}_{f m}(0)$ and $\boldsymbol{\mu}_{m i}(0)$ by $\boldsymbol{\mu}_{f m}$ and $\boldsymbol{\mu}_{m i}$. The expression of the rate of the transition can be rewritten as

$$
\begin{aligned}
W_{i \rightarrow f}^{(2)}(\omega)= & \frac{\pi}{8 \hbar^{4}}\left|M_{i f}(\omega)\right|^{2} \sum_{v} \sum_{v^{\prime}} P_{i v}\left|\left\langle\Theta_{f v^{\prime}} \mid \Theta_{i v}\right\rangle\right|^{2} \\
& \times D\left(\omega_{f v^{\prime}, i v}-2 \omega, \gamma_{f v^{\prime}, i v}\right)
\end{aligned}
$$

where $M_{i f}(\omega)$ denotes the two-photon transition strength

$$
\begin{aligned}
M_{i f}(\omega) & =\sum_{m} \frac{\left(\boldsymbol{\mu}_{f m} \cdot \mathbf{E}_{0}\right)\left(\boldsymbol{\mu}_{m i} \cdot \mathbf{E}_{0}\right)}{\omega_{m i}-\omega} \\
& =\mathrm{e}^{2}\left|\mathbf{E}_{0}\right|^{2} \sum_{m} \frac{\left(\mathbf{R}_{f m} \cdot \lambda\right)\left(\mathbf{R}_{m i} \cdot \lambda\right)}{\omega_{m i}-\omega} \\
& =\mathrm{e}^{2}\left|\mathbf{E}_{0}\right|^{2} S_{f i}^{\lambda}(\omega)
\end{aligned}
$$

Here, the term

$$
S_{f i}^{\lambda}(\omega)=\sum_{A}^{X, Y, Z} \sum_{a}^{x, y, z} \xi_{A a} \lambda_{A}^{2} \sum_{m} \frac{\mathbf{R}_{f m}^{a} R_{m i}^{a}}{\omega_{m i}-\omega}
$$

is a transformation two-photon transition matrix element tensor, which projects the transition dipole moments from the molecular frame $(x, y$, and $z)$ onto the laboratory frame $(X$, $Y$, and $Z$ ) through the transformation matrixes $\xi_{A a}$ s. In eq 15 , the vibrational levels are not included in the calculation, assuming that the energy gap between the electronic states is much larger than that between the pure vibrational states, i.e., the Placzek approximation. Taking the orientational average on the square of the transformation matrix element yields ${ }^{47-49}$

$$
\left\langle\left|S_{f i}^{\lambda}(\omega)\right|^{2}\right\rangle=\frac{1}{3} \sum_{a}^{x, y, z}\left|\sum_{m} \frac{\mathbf{R}_{f m}^{a} \mathbf{R}_{m i}^{a}}{\omega_{m i}-\omega}\right|^{2}
$$

It follows that, in the low-temperature case, the orientationaveraged 2PA transition rate can be written as

$$
\begin{aligned}
\left\langle W_{i \rightarrow f}^{(2)}(\omega)\right\rangle= & \frac{\pi \mathrm{e}^{4}\left|\mathbf{E}_{0}\right|^{4}}{24 \hbar^{4}} \sum_{a}^{x, y, z}\left|\sum_{m} \frac{\mathbf{R}_{f m}^{a} \mathbf{R}_{m i}^{a}}{\omega_{m i}-\omega}\right|^{2} \\
& \times \sum_{v^{\prime}}\left|\left\langle\Theta_{f v^{\prime}}, \Theta_{i 0}\right\rangle\right|^{2} D\left(\omega_{f v^{\prime}, i 0}-2 \omega, \gamma_{f v^{\prime}, i 0}\right)
\end{aligned}
$$

The corresponding 2PA cross-section becomes

$$
\begin{aligned}
\delta_{i \rightarrow f}(\omega)= & 2 \frac{\left\langle W_{i \rightarrow f}^{(2)}(\omega)\right\rangle(\hbar \omega)^{2}}{I^{2}} \\
= & \frac{16 \pi^{3} \omega^{2}}{3 c \hbar^{2}} \sum_{a}^{x, y, z}\left|\sum_{m} \frac{\mathbf{R}_{f m}^{a} \mathbf{R}_{m i}^{a}}{\omega_{m i}-\omega}\right|^{2} \\
& \times \sum_{v^{\prime}}\left|\left\langle\Theta_{f v^{\prime}} \mid \Theta_{i 0}\right\rangle\right|^{2} D\left(\omega_{f v^{\prime}, i 0}-2 \omega, \gamma_{f v^{\prime}, i 0}\right)
\end{aligned}
$$

where $I$ is the light intensity

$$
I=\frac{c}{8 \pi}\left|\mathbf{E}_{0}\right|^{2}
$$

3.3. Computational Methods. In this article, the Becke's three-parameter exchange functional in combination with the LYP correlation functional (B3LYP) and the standard 6$31 \mathrm{G}(\mathrm{d}, \mathrm{p})$ basis set were used to optimize the ground state molecular equilibrium geometry. Then, the excited states were calculated using both TD-DFT (TD-BPE0/6-31G(d,p)) and ZINDO method based on the B3LYP optimized ground state molecular. The UV-vis spectra were calculated using the transition dipole moment from the ground state to the excited state obtained from both TD-BPE0 and ZINDO method calculations. Since the transition dipole moments between excited states could not be obtained by TD-BPE0 calculation, 2PA properties were calculated with ZINDO calculation. The excited-state properties of the five molecules (chromophores 1 to 5) were characterized and investigated with the threedimensional cube representation of the charge difference density (CDD), ${ }^{33,50-52}$ which shows the distribution of net change in electron and hole densities as a result of the electronic transitions and the orientation of the possible ICT states. All computations were performed with the Gaussian03 program package. ${ }^{53}$

\section{RESULTS AND DISCUSSION}

4.1. UV-Vis Absorption and Fluorescence Spectra. The major building blocks of chromophores $1-5$ are the electron-donating (D) triphenylamine and the accepting (A) benzothiadiazole moieties. All the chromophores 1-5 show two intense absorption bands in the near-UV in the region of $300-400 \mathrm{~nm}$ and visible spectral regions from 450 to $600 \mathrm{~nm}$ (shown in Figure 1), corresponding to the transitions from ground state to the higher excited states and low-lying ICT states, respectively. ${ }^{12,28,54-56}$ It is found that the increase of quadrupolar unit branches in the molecules leads to a slight red-shift of both the absorption and emission bands (Figure 1). This is an indication of interactions between the moieties, resulting in charge redistribution and extended delocalization. ${ }^{31,59}$ From the line shape of the observed absorption, it is found that the interaction between branches are not so significant to split the low-lying ICT absorption band.

The absorption and emission spectra of chromophore $\mathbf{4}$ are significantly red-shifted from the other chromophores. This is 


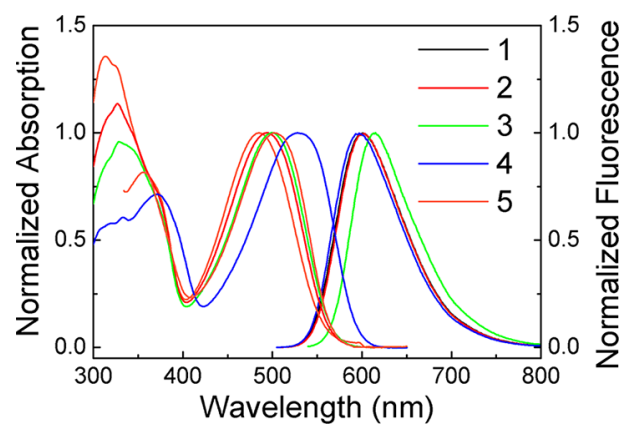

Figure 1. Normalized absorption and fluorescence spectra of chromophores $\mathbf{1 - 5}$ in toluene.

analogous to the phenomena reported by Nicolle et. al, ${ }^{57}$ where the introduction of a $\mathrm{C}=\mathrm{C}$ bond in chromophore 4 leads to a larger red shift and absorption enhancement, compared to that of $\mathrm{C}-\mathrm{C}$ in chromophore 3 and $\mathrm{C} \equiv \mathrm{C}$ in chromophore 5. For chromophore 4, both the absorption and emission of low-lying ICT band exhibit a red shift of 30 and $13 \mathrm{~nm}$ with respect to the chromophore 3 , respectively, whereas, chromophore 5 exhibits only a small red shift of the low-lying ICT states absorption band with respect to the chromophore 3 . Surprisingly, the emission of chromophore 5 shows a slight blue shift compared to that of chromophore 3 . The explanation for the unusual phenomena in chromophore $\mathbf{5}$ is that the sp and $\mathrm{sp}^{2}$ hybridization of the ethynyl results in poorer $\pi$-orbital overlap and mismatched orbital energy. ${ }^{18}$ In addition, as a $\pi$ spacer, $\mathrm{C} \equiv \mathrm{C}$ introduces more $\pi$ electrons to the delocalized system than $\mathrm{C}=\mathrm{C}$ does, but because of the absence of steric hindrance and the very low rotational barrier about $0.9 \mathrm{kcal} /$ mol per cylindrical triple bond, the twisting about the cylindrical triple bond in chromophore 5 interrupts the conjugation around $\mathrm{C} \equiv \mathrm{C}$ on the planarity of the conjugated system, resulting in a relatively higher ICT state energy compared to the large conjugation through $\mathrm{C}=\mathrm{C}$ with lower ICT state energy level, correspondingly. ${ }^{10,14,58}$ Furthermore, unlike $\mathrm{C} \equiv \mathrm{C}$ and $\mathrm{C}-\mathrm{C}$ with low rotational barriers, the rigid $\mathrm{C}=\mathrm{C}$ conjugates the linear structure of branches at a certain degree, thereby increasing the electrostatic coupling between the dipolar branches. ${ }^{26}$ This may be another reason for the larger spectral red shift observed in chromophore 4. Goodson et al. also showed that, for the $\mathrm{D}-\pi-\mathrm{A}-\pi-\mathrm{D}$ structure, replacement of a single bond by a double bond led to a larger red shift both in absorption and fluorescence than that by triple bond, ${ }^{18,54,59}$ which is consistent with our experimental results. Moreover, similar phenomenon has also been observed in our previous study in which the branched chromophore, which formed by a simple unconjugated combination of two or three $\mathrm{D}-\pi-\mathrm{A}-\pi-\mathrm{D}$ moieties, showed only very small alteration in spectral features compared to the monomers, ${ }^{12,28}$ whereas the chromophore formed by conjugated moieties, which have $\pi$ bridge linkers, usually have a remarkable delocalized ICT state, and therefore, significantly different spectral features from those of isolated moieties. ${ }^{12,28}$ As the shift of the absorption and emission band is not significant except for chromophore 4, we conclude that, in chromophores $1,2,3$, and 5, the linkage of moieties do not conjugate well with the branched moieties, and each moiety keeps its original electronic state feature partly. The spectral parameters of chromophores 1-5 are summarized in Table 1. More details on calculated spectral parameters of chromophores 1-5 are listed in Table S1 (see Supporting
Table 1. Photophysical Data of Chromophores $1-5^{a}$

\begin{tabular}{llllll} 
photophysical data & \multicolumn{1}{c}{} & $\mathbf{2}$ & \multicolumn{1}{c}{$\mathbf{3}$} & $\mathbf{4}$ & $\mathbf{5}$ \\
$\lambda_{\text {abs }}(\mathrm{nm})$ & 485 & 494 & 500 & 528 & 502 \\
$\lambda_{\text {em }}(\mathrm{nm})$ & 592 & 601 & 602 & 614 & 597 \\
$\lambda_{1,0}^{\text {ZNDO }}(\mathrm{nm})$ & 484 & 489 & 490 & 503 & 519 \\
$\mu_{1,0}^{\text {ZINDO }}(\mathrm{D})$ & 7.9 & 9.9 & 9.9 & 11.2 & 11.9 \\
$\lambda_{1,0}^{\text {TDDDFT }}(\mathrm{nm})$ & 571 & 602 & 609 & 653 & 622 \\
$\mu_{1,0}^{\text {TD-DFT }}(\mathrm{D})$ & 10.4 & 13.3 & 12.7 & 15.9 & 15.9 \\
$\tau_{\text {em }}(\mathrm{ns})$ & 6.7 & 6.3 & 6.6 & 3.4 & 5.2 \\
slope $\left(\mathrm{cm}^{-1}\right)$ & 6685 & 5965 & 6271 & 5952 & 7419 \\
$a_{0}(\AA)$ & 7.03 & 8.06 & 8.39 & 8.82 & 9.16 \\
$\Delta \mu(\mathrm{D})$ & 15.3 & 17.7 & 19.4 & 20.3 & 23.9
\end{tabular}

${ }^{a} \lambda_{\text {abs }}$ and $\lambda_{\text {em }}$ are absorption and emission wavelengths measured in toluene, respectively. $\lambda_{1,0}^{\mathrm{ZINDO}}$ and $\lambda_{1,0}^{\mathrm{TD}-\mathrm{DFT}}$ are calculated absorption wavelengths for transitions from the ground state to the first excited state by using ZINDO and TD-DFT methods, respectively, while $\mu_{1,0}^{\mathrm{ZINO}}$ and $\mu_{1,0}^{\mathrm{TD}-\mathrm{DFT}}$ are the corresponding amplitudes of the transition dipole moments. $\tau_{\mathrm{em}}$ is the fluorescence lifetime, excited at $465 \mathrm{~nm}$ and detected at $600 \mathrm{~nm}$. Slope is obtained from Figure 2 by fitting the Lippert-Mataga relationship between the experimental results of Stokes shift and the orientational polarizability of solvents. The Onsager cavity radii $\left(a_{0}\right)$ was estimated from quantum chemical calculation by using DFT method under the B3LYP/6-31 $\mathrm{g}^{\text {*** }}$ level. ${ }^{60,61} \Delta \mu$ is the difference between the excited state and ground state dipole moments. The positive $\Delta \mu$ means that the dipole moment of the emitted state is larger than that of the ground state.

Information). It is found that the calculated energy gaps for all the compounds are very close to the experimental values obtained from the UV-vis absorption spectra (see Figure 1). The slight discrepancy between the calculated and experimental values results from the solvation effects, which are not accounted for in the gas-phase calculations.

4.2. Solvation Effects. In order to understand the solvent effects on the nature of optical excitations and the degree of ICT as well as polar nature of the excited state for the compounds 1-5, we correlate the Stokes shift in a series of solvents. Chromophores $\mathbf{1 - 5}$ show a nearly identical response on solvent polarity, i.e., absorption spectra exhibits less dependence on solvent alteration, on the contrary, fluorescence shows a remarkable red-shift upon increasing solvent polarity. Figure 2a shows the normalized absorption and emission spectra of chromophore 3 in different polar solvents. Stokes shifts increase with increasing solvent polarity, which is consistent with the stabilization of the polar excited states (CT states) by the polar solvents. The obvious absorption change around $350 \mathrm{~nm}$ in methanol is probably due to the specific interaction between the solute chromophore and the solvent molecule because methanol is the only protic solvent among the solvents used here. ${ }^{62}$

Furthermore, the solvatochromic behavior of the chromophores follows the Lippert-Mataga relationship: ${ }^{63}$

$$
\Delta \nu=\tilde{\nu}_{\mathrm{abs}}-\tilde{\nu}_{\mathrm{em}}=\frac{2 \Delta \mu^{2}}{h c a^{3}} \Delta f+\text { const }
$$

where $\tilde{\nu}_{\text {abs }}$ and $\tilde{\nu}_{\text {em }}$ are the wavenumbers of the absorption and fluorescence maxima, $h$ is the Planck's constant, $c$ is the speed of light, $a$ is the radius of the solute spherical cavity, and $\Delta f$ is the orientational polarizability of solvent, which can be defined as

$$
\Delta f=\frac{(\varepsilon-1)}{(2 \varepsilon+1)}-\frac{\left(n^{2}-1\right)}{\left(2 n^{2}+1\right)}
$$



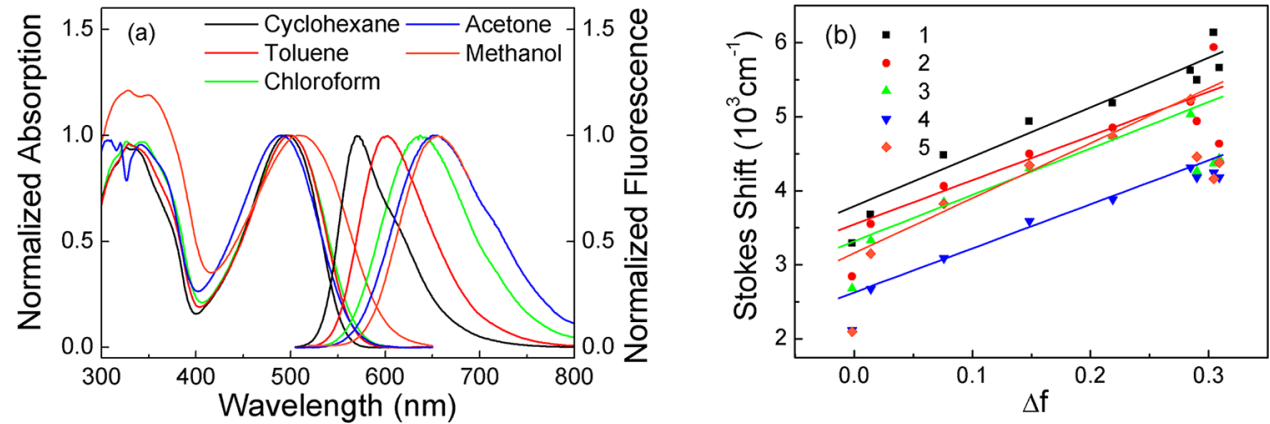

Figure 2. Solvatochromic behavior of chromophore $\mathbf{3}$ in different solvents (a) and Lippert-Mataga correlations for chromophores 1-5 between the Stokes shifts and solvent polarities (b).
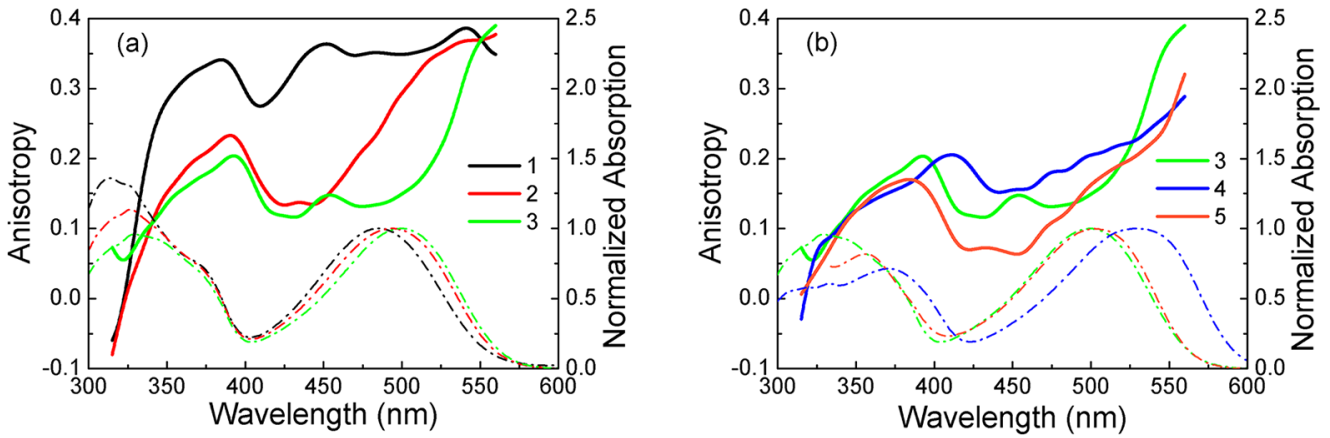

Figure 3. Fluorescence excitation anisotropy spectra (solid lines) of chromophores 1-3 (a) and 3-4 (b) in polystyrene film measured by monitoring the emission at $620 \mathrm{~nm}$. Normalized absorption spectra (dashed lines) are also shown for comparison.

where $\varepsilon$ is the dielectric constant, and $n$ is the refractive index of the solvent.

Figure $2 \mathrm{~b}$ shows the Lippert--Mataga relationship between the experimental results of Stokes shift and the orientational polarizability of solvents. It is found that solvent polaritydependent Stokes shifts of all the five chromophores show nearly identical behaviors. Chromophore 4 strays away a little, indicating stronger branch interaction in chromophore 4. To quantitatively determine the polarity of the excited ICT state, we also examined the dipole moment change $(\Delta \mu)$ between the excited state and ground state dipole moments based on the eqs 23 and 24 (as shown in Table 1).

4.3. Transition Dipole Moment and Excited-State Localization. From the normalized absorption spectrum and fluorescence spectrum shown above, it seems that multibranched compounds with quadrupolar $\mathrm{D}-\pi-\mathrm{A}-\pi-\mathrm{D}$ structure have almost identical spectral features with those of the monomer counterpart. ${ }^{12,18,27,28,34}$ Excited state energy redistribution among the branches is thought to be a common feature in interacting multibranched systems studied here. One of the important interactions of the redistribution is excitation delocalization/localization; the strength of interaction depends on the molecular structure with positions of donor/acceptors. Both symmetry-breaking phenomena in excited state (caused by vibrational relaxation and solvation) and disorder-induced symmetry lowering (caused by ground state solute-solvent interaction) have been reported to contribute to the excitation localization. ${ }^{25-27,42-44,64,65}$ In order to track down the excited state energy redistribution and the interbranch interaction of the multibranched molecules, it is instructive to determine the direction of molecular transition dipole moment and polarization of the spectrum.
TD-DFT calculations could provide us the theoretical prediction of the features of these excited states and the transition dipole moments from the ground state to excited states. The calculated intramolecular charge-transfer (ICT) nature of the electronic transitions from the ground state to the lowest excited states are clearly illustrated in Table S2 (Supporting Information) by the charge difference density (CDD) patterns. In these transitions, charge density shifts mostly from the electron-donating (D) triphenylamine moieties to the accepting (A) benzothiadiazole moieties. The quadrupolar feature of chromophore $\mathbf{1}$ is clearly shown in Table S2a (Supporting Information). Chromophore $\mathbf{1}$ has two wide separated ICT states (high-energy ICT state $\left|2_{1 \mathrm{qq}}\right\rangle$ and lowenergy ICT state $\left.\left|1_{1 q}\right\rangle\right)$ in the broad region above $400 \mathrm{~nm}$, similar to our previous results, ${ }^{12}$ where the energy transfer occurs from high-energy ICT to the low-energy ICT states. According to the Frenkel exciton model, the excited states of multibranched molecules can be constructed from these two ICT states of chromophore 1. For example, in two-branched chromophore $2,\left|1_{1 q}\right\rangle$ is split into $\left|1_{2 q}\right\rangle$ and $\left|2_{2 q}\right\rangle$, and $\left|2_{1 q}\right\rangle$ is split into $\left|3_{2 q}\right\rangle$ and $\left|4_{2 q}\right\rangle$; while in three-branched chromophores, $\left|1_{1 \mathrm{q}}\right\rangle$ is split into $\left|1_{3 \mathrm{q}}\right\rangle,\left|2_{3 \mathrm{q}}\right\rangle$, and $\left|3_{3 \mathrm{q}}\right\rangle$, and $\left|2_{1 \mathrm{q}}\right\rangle$ is split into $\left|4_{3 q}\right\rangle,\left|5_{3 q}\right\rangle$, and $\left|6_{3 q}\right\rangle$ (see Scheme 2). The relevant expressions of excited states of multibranched molecules are listed in Table S2 (Supporting Information). The predictions of the Frenkel exciton model are in qualitative agreement with the CDD patterns, the location of the excited states, and the direction of transition dipole moments calculated from TDDFT.

4.4. Fluorescence Excitation Anisotropy Spectra. From the CDD pattern as discussed in the previous sections, it is found that, for the low-energy ICT states, triphenylamine core, where the holes are mainly located after excitation, is the most 
active donor; while for high-energy ICT states, triphenylamine at the end of each branch is more active. Thus, by measuring the fluorescence excitation anisotropy spectra, we could figure out the model of the excited state progression between the high-energy and low-energy ICT states. It is found that, when the multibranched chromophores 2-5 were excited to the high-energy ICT states, the electron transfers from the end of each branch to the triphenylamine core are prior to the fluorescence emission.

Fluorescence excitation anisotropy spectra provided us a measuring result of the angle between the absorption and emission transition dipole moments. We performed fluorescence excitation anisotropy measurements of the five compounds in an isotropic polystyrene film at room temperature, in order to avoid the fast rotation of molecules during excitation and fluorescence. Figure 3 shows the excitation anisotropy spectra of chromophores 1-5 measured by monitoring the emission from the lowest ICT state at 620 $\mathrm{nm}$. For comparison, normalized absorption spectra of chromophores $\mathbf{1 - 5}$ in toluene are also shown.

As shown in Figure 3, the fluorescence excitation anisotropy spectra of chromophores 1-5 show qualitatively different behavior. Herein, we pay more attention to the anisotropy of the high-energy and low-energy ICT bands that dominate the fluorescence properties. The anisotropy of chromophore $\mathbf{1}$ is relatively constant in the range of low-energy ICT state from 450 to $550 \mathrm{~nm}$ with the value of $r=0.35$, close to the theoretical maximum of 0.4. This is in accordance with the character of linear chromophores that have collinear transition moments for the absorption and emission. ${ }^{66-68}$ Furthermore, the angle between absorption and emission transition moment can be approximately calculated using eq $25:^{66,67}$

$$
r=\frac{2}{5}\left(\frac{3 \cos ^{2} \beta-1}{2}\right)
$$

In vitrified PS film, the molecule rotational diffusion was ruled out, thus a larger angle between the absorption and emission transition moment indicates a substantial redistribution of the excitation energy prior to emission. ${ }^{34,59}$

For chromophore 1, $r=0.35$ corresponds to the $\beta=17^{\circ}$ between the absorption and emission dipoles. Since there is no interbranch interaction in chromophore 1, this small angle between absorption and emission transition moment $\left(\beta=17^{\circ}\right)$ represents the molecular structure distortion caused by disorder.

As discussed in the previous section, there are two different ICT excited states (high-energy $\left(\left|2_{1 q}\right\rangle\right)$ and low-energy $\left(\left|1_{1 q}\right\rangle\right)$ ICT states) located at different excitation wavelengths in the region from 400 to $600 \mathrm{~nm}$. However, the transition dipole moments from ground state to $\left|1_{1 q}\right\rangle$ or $\left|2_{1 q}\right\rangle$ are in different directions because the donor and two acceptor groups are not strictly straight as shown in Scheme 2. The angle between the transition dipole moments from the ground state to $\left|1_{1 \mathrm{q}}\right\rangle$ and $\mid$ $\left.2_{1 \mathrm{q}}\right\rangle$ is around $28^{\circ}$ (corresponding to an anisotropy value of $r \approx$ 0.27 at $420 \mathrm{~nm}$ ). When chromophore 1 is excited at $420 \mathrm{~nm}$ from the ground state to $\left|2_{1 q}\right\rangle$ where the high-energy ICT band is located, the energy was immediately transferred to the lowenergy ICT state $\left(\left|1_{1 q}\right\rangle\right)$ from $\left|2_{1 q}\right\rangle$ before the emission, leading to a decreased anisotropy value, while a low anisotropy value of about 0.27 is observed around $420 \mathrm{~nm}$ for chromophore 1 as shown in Figure 3a.
For chromophores 2 and $\mathbf{3}$ as shown in Figure 3a, the anisotropy value $r$ of about 0.13 is observed at the high-energy ICT side around $420-440 \mathrm{~nm}$, corresponding to the displacement angle of $\beta \approx 43^{\circ}$, which is much larger than $\sim 27^{\circ}$ of the monomer chromophore 1 in the same wavelength region. The anisotropy value gradually increases as the excitation energy decreases and then reaches an anisotropy larger than 0.30 around $550 \mathrm{~nm}$ in the low energy ICT region. This indicates that significant redistribution of the excitation energy among the split high-energy and low-energy ICT states occurs in chromophores $\mathbf{2}$ and $\mathbf{3}$, respectively, prior to emission. Since the depolarization of the emission through rotational diffusion of molecules itself is strongly suppressed by the dry polystyrene film, an intramolecular excitation energy transfer mechanism from high-energy to low-energy ICT states should be involved in explaining the observed depolarization. ${ }^{12,33,66,69,70}$

By taking a closer look at the anisotropy spectra of chromophores $\mathbf{2}$ and $\mathbf{3}$ from Figure $3 \mathrm{a}$, it is found that, similarly with chromophore 1 , anisotropy values are relatively low in the region from 400 to $440 \mathrm{~nm}$. As shown in Scheme 2 and Table S2 (Supporting Information), in this region, the second excited state $\left|2_{1 q}\right\rangle$ is split into $\left|3_{2 q}\right\rangle$ and $\left|4_{2 q}\right\rangle$ for chromophore 2 , and into $\left|4_{3 q}\right\rangle,\left|5_{3 q}\right\rangle$, and $\left|6_{3 q}\right\rangle$ for chromophore 3. $\left|3_{2 q}\right\rangle$ and $\left|4_{2 q}\right\rangle$ are perpendicular to each other, and $\left|4_{3 q}\right\rangle, \mid$ $\left.5_{3 q}\right\rangle$, and $\left|6_{3 q}\right\rangle$ are vertical to each other. Thus, as a result of the excited state splitting, anisotropy values of chromophores $\mathbf{2}$ and 3 (0.12 and 0.14) are even lower than that of chromophore 1 when energy transfer occurs from the high energy ICT state around $400 \mathrm{~nm}$ to low energy ICT state around $550 \mathrm{~nm}$. Furthermore, in the region above $450 \mathrm{~nm}$, as shown in Figure 3, it is found that the anisotropy value of chromophore $\mathbf{2}$ increases faster with the increasing excitation wavelengths than that of chromophore 3 . It is reasonable that, the first excited state $\left|1_{1 q}\right\rangle$ is split into $\left|1_{2 q}\right\rangle$ and $\left|2_{2 q}\right\rangle$ for chromophore 2 , and into $\left|1_{3 q}\right\rangle$, , $\left.2_{3 q}\right\rangle$, and $\left|3_{3 q}\right\rangle$ for chromophore 3 with orthogonal transition dipole moments with respect to each other from the ground state (shown in Scheme 2). Therefore, as the excitation energy decreases, the lowest $\left|1_{2 q}\right\rangle$ state mainly contributes to the absorption spectra as the final emission state, the anisotropy value of chromophore $\mathbf{2}$ increase quickly since energy transfer only occurs between two low-energy ICT states. Since chromophore 3 has a different symmetry character from chromophore 2 , the energies of the degenerate lowest excited states of chromophore $3,\left|1_{3 q}\right\rangle$ and $\left|2_{3 q}\right\rangle$ are slightly different because of the disorder and steric effects, the energy transfer occurs between three split ICT states in the low-energy ICT region. Therefore, the anisotropy value increases slowly with increasing excitation wavelength. Therefore, from 450 to 520 $\mathrm{nm}$, unlike the fast increase in anisotropy of chromophore 2, the anisotropy value of chromophore 3 remains low $(\sim 0.13)$ before the final emission state. Anyway, the energy levels of I $\left.1_{3 q}\right\rangle$ and $\left|2_{3 q}\right\rangle$ are not fully degenerated because the disorderinduced symmetry-breaking of the molecule in solution further lowers the excitation energy of $\left|1_{3 q}\right\rangle$ and makes it as the final emission state of the absorption at the red edge of the absorption (above $520 \mathrm{~nm}$ ). Therefore, the anisotropy value of chromophore 3 finally increases above $520 \mathrm{~nm}$ up to 0.35 . Furthermore, as shown in Figure $3 b$, it is also found that there are some differences in the anisotropy values for chromophores 3,4 , and 5 in the region $400-450 \mathrm{~nm}$, where the absorption at $400-450 \mathrm{~nm}$ results from the high-energy ICT states of the chromophores 3-5. The angle between transition dipole 
moments from the ground state to the low-energy ICT states and those from the ground state to the high-energy ICT states determines the anisotropy value of in this region.

Similar to chromophore 3 , chromophores $\mathbf{4}$ and $\mathbf{5}$ are also of three quadrupolar excitons with $C_{3 h}$ symmetry. The anisotropy spectra of them are generally similar to that of chromophore 3 (as shown in Figure $3 \mathrm{~b}$ ). They all have low anisotropy value in the region of the local excited (LE) states. In the regions below $400 \mathrm{~nm}$, chromophores $3-5$ even have almost the same anisotropy value. However, the difference of the anisotropy values between chromophores $3-5$ in the region of 400-510 $\mathrm{nm}$ is noticeable. Chromophore $\mathbf{4}$ has the highest anisotropy value of the three chromophores, while chromophores 3 and 5 show the lower anisotropy values. The observed difference in anisotropy results from the torsional disorder and symmetry of the structures of these branched chromophores, which lead to the nondegenerate energy levels. ${ }^{27,44,71}$ The energy transfer then occurs among these nondegenerated ICT states, which results in a large decrease of anisotropy value during energy transfer.

By taking a closer look at the CDD patterns of multibranched chromophores (chromophores 2-5) as listed in Table S2 (Supporting Information), it is found that, for the high-energy ICT states, the excitation is mostly attributed to the electron-transfer from triphenylamine moieties at the end of each branch to the benzothiadiazole moieties, whereas for the low-energy ICT states, the excitation is mostly attributed to the electron-transfer from triphenylamine core to the benzothiadiazole moieties. Therefore, for low-energy ICT states, triphenylamine core is the most active donor, the throughbond interaction between branches via the triphenylamine core are strong, and the CDD distribution on each branch shows high symmetry with the overall molecule charge distribution. On the contrary, for high-energy ICT states, triphenylamines at the end of each branch are more active, and the connection between branches are interrupted, and the charge transfer states are located within each branch, unlike that of low-energy ICT states with the distribution of the electrons over all branched molecules; thus, CDD distribution at each branch is distributed unequally. The obvious evidence for the difference between low- and high-energy ICT states is clearly seen in the CDD pattern of chromophore $\mathbf{2}$ in Table S2b (Supporting Information).

Regarding three-branched chromophores such as chromophores 3-5 with ideal $C_{3 h}$ symmetry, the interaction among branches should be very strong, and charge transfer after excitation among branches is highly delocalized. In this case, the CDD distribution on each branch would agree very well with the expressions of the $C_{3 h}$ symmetry Frenkel exciton model for low-energy ICT states

$$
\begin{aligned}
& \left|1_{3 q}\right\rangle=\frac{1}{\sqrt{2}}\left(\left|1_{q(b)}\right\rangle-\left|1_{q(c)}\right\rangle\right) \\
& \left|2_{3 q}\right\rangle=\frac{1}{\sqrt{6}}\left(2\left|1_{q(a)}\right\rangle-\left|1_{q(b)}\right\rangle-\left|1_{q(c)}\right\rangle\right) \\
& \left|3_{3 q}\right\rangle=\frac{1}{\sqrt{3}}\left(\left|1_{q(a)}\right\rangle+\left|1_{q(b)}\right\rangle+\left|1_{q(c)}\right\rangle\right)
\end{aligned}
$$

and high-energy ICT states

$$
\left|4_{3 \mathrm{q}}\right\rangle=\frac{1}{\sqrt{3}}\left(\left|2_{\mathrm{q}(\mathrm{a})}\right\rangle+\left|2_{\mathrm{q}(\mathrm{b})}\right\rangle+\left|2_{\mathrm{q}(\mathrm{c})}\right\rangle\right)
$$

$$
\begin{aligned}
& \left|5_{3 \mathrm{q}}\right\rangle=\frac{1}{\sqrt{2}}\left(\left|2_{\mathrm{q}(\mathrm{b})}\right\rangle-\left|2_{\mathrm{q}(\mathrm{c})}\right\rangle\right) \\
& \left|6_{3 \mathrm{q}}\right\rangle=\frac{1}{\sqrt{6}}\left(2\left|2_{\mathrm{q}(\mathrm{a})}\right\rangle-\left|2_{\mathrm{q}(\mathrm{b})}\right\rangle-\left|2_{\mathrm{q}(\mathrm{c})}\right\rangle\right)
\end{aligned}
$$

For chromophores 3-5, the connection of benzothiadiazole to triphenylamine at the end of the branches are identical, while the linkage of benzothiadiazole and the triphenylamine core are different. Comparing the difference in structures between chromophores 3-5, the effect of through-bond interaction on the excited state features could be evaluated by considering the conjugation of $\pi$-linkers.

The significant red-shift of the absorption band in chromophore 4 spectra indicates that the interbranch interaction is strong. As the sizes of chromophores 3-5 are almost the same and the distance between the branches are similar in all of these molecules, the obviously strong interbranch interaction of chromophore 4 is attributed to the through-bond interaction caused by rigid $\mathrm{C}=\mathrm{C}$ linkage.

Because of the rigid and conjugated $\mathrm{C}=\mathrm{C}$ in chromophore 4, charge redistribution among branches is very efficient. Both the low-energy ICT states and the high-energy ICT states have similar delocalization features, and the CDD distribution at each branch agrees very well with the expressions of the high $C_{3 h}$ symmetry Frankel exciton model (see Table S2d in Supporting Information). Thus, the orientations of the transition dipole moments from the ground state to lowenergy ICT states and that from the ground state to highenergy ICT states are almost the same with the angle between them about $\sim 6.9^{\circ}$ from the calculated results, but two orthogonally polarized degenerate excited states, whose degeneracy is slightly removed because of structure disorder and solvation, leading to a large anisotropy plateau value in the high-energy ICT side, and an almost plateau anisotropy spectrum of chromophore 4 from 400 to $550 \mathrm{~nm}$ is observed as shown in Figure 3b.

On the contrary, if the conjugation of the triphenylamine core and three branches are interrupted, the $C_{3 h}$ symmetry feature of the excited states would easily be broken. In chromophore 3, $\mathrm{C}-\mathrm{C}$ between triphenylamine and benzothiadiazole is twisted by the steric effect. As a result, the CDD distribution of both low- and high-energy ICT states do not agree with the expressions of the $C_{3 h}$ symmetry Frenkel exciton model (see Table S2c in Supporting Information). When the excitation is localized in a certain branch (or two branches), the angles between transition dipole moments from the ground state to the low-energy ICT states and those from the ground state to the high-energy ICT states are estimated to be $\sim 19.3^{\circ}$. As a result, the excitation is efficiently localized, together with degeneracy of the two orthogonally polarized degenerate excited states strongly removed because of structure disorder and solvation, ${ }^{26}$ leading to the decreased anisotropy value in the high-energy ICT side as shown in Figure $3 .{ }^{13,66}$

Regarding chromophore 5, conjugation among the triphenylamine core and three branches through $\mathrm{C} \equiv \mathrm{C}$ do exist, but it is not as strong as that in chromophore 4 because of the small twisting barrier of the $\mathrm{C} \equiv \mathrm{C}$ bond. ${ }^{10,58}$ Therefore, in chromophore 5, for low-energy ICT states, triphenylamine core is more active, charge is partially distributed overall molecule, and the CDD distribution at each branch agrees with $C_{3 h}$ symmetry Frenkel exciton model expressions (see Table S2e in Supporting Information), while for high-energy ICT 
states, triphenylamine core is not that active, charge redistribution is partially restricted, and the CDD distribution is slightly different from the expressions of an ideal $C_{3 h}$ symmetry Frenkel exciton model. The angle between transition dipole moments from the ground state to the low-energy ICT states and those from the ground state to the high-energy ICT states is estimated about $\sim 19.3^{\circ}$. However, it has been found in our previous studies that the twisting barrier around $\mathrm{C} \equiv \mathrm{C}$ is too low to maintain the relatively strong conjugation ${ }^{10,58}$ and that the $C_{3 h}$ symmetry is easily broken in chromophore 5 and the degeneracy of the two orthogonally polarized degenerate excited states are largely removed, which leads to the decreased anisotropy value as shown in Figure $3 b$.

4.5. Two-Photon Absorption. According to measured absorption spectra (as shown in Figure 1) and the TD-DFT calculation results (see Table S1 in Supporting Information) for all the studied chromophores, the one-photon absorptions (1PA) of the low-energy ICT states have strong oscillator intensities with large oscillator strengths, whereas the onephoton absorptions (1PA) of the high-energy ICT states is very weak with small oscillator intensity and is hidden in the broad and strong absorptions of low-energy ICT states. Hence, the optical properties of the high-energy ICT states are therefore difficult to be identified from 1PA measurements because of the very weak 1PA absorption. Although the position of highenergy ICT states could be roughly evaluated by fluorescence excitation anisotropy spectra discussed above, as the complementary measurement of 1PA property, two-photon absorption measurements are better suited to determine the detailed spectral properties of the high-energy ICT states.

As described in eq 21,2PA and 1PA have the same FranckCondon factor $\sum_{v^{\prime}}\left|\left\langle\Theta_{f v^{\prime}} \mid \Theta_{i 0}\right\rangle\right|^{2}$; thus, 2PA and 1PA spectra should have the same vibronic band shape. ${ }^{46,72}$ Since the selection rule is different for two-photon absorption from the one-photon absorption, ${ }^{13,72}$ two-photon absorption is one of the most important nonlinear optical properties of organic charge-transfer molecules, which is strongly dependent on the molecular structure, such as electron donor and acceptor features, $\pi$-bridge group and length, geometric configuration, etc. $^{11,73-78}$ Therefore, 2PA spectrum provides us complementary information about excited states inaccessible via onephoton absorption. $^{46}$

Figure 4 shows the experimental and calculated two-photon absorption and one-photon absorption spectra of chromophores 1-5. It is found that the strong 1PA absorption band in the $450-600 \mathrm{~nm}$ spectral range is mainly attributed to the transition from the ground state to the lowest ICT states, where the one-photon absorption of the high-energy ICT state is very weak, which is hidden in the strong 1PA spectra of the lowenergy ICT states. Oppositely, the strong absorption band of 2PA spectra in $770-870 \mathrm{~nm}$ (385-435 $\mathrm{nm}$ in 1PA spectra) is attributed to the transition from the ground state to the highenergy ICT states. Because of the limited wavelength region of the femtosecond laser used in our 2PA experiments, 2PA spectra related to the low-energy ICT state in the region above $950 \mathrm{~nm}$ could not be obtained. The 2PA cross-sections in lowenergy ICT states are much smaller compared to those of highenergy ICT states as shown by the calculated results of Figure 4 and also reported for other similar multibranched quadrupolar molecules. ${ }^{25-27,42,44}$

To fully understand the 2PA properties of the chromophores 1-5, we turn to describe the structure-dependent 2PA spectra theoretically. Table S1 (Supporting Information) lists all the

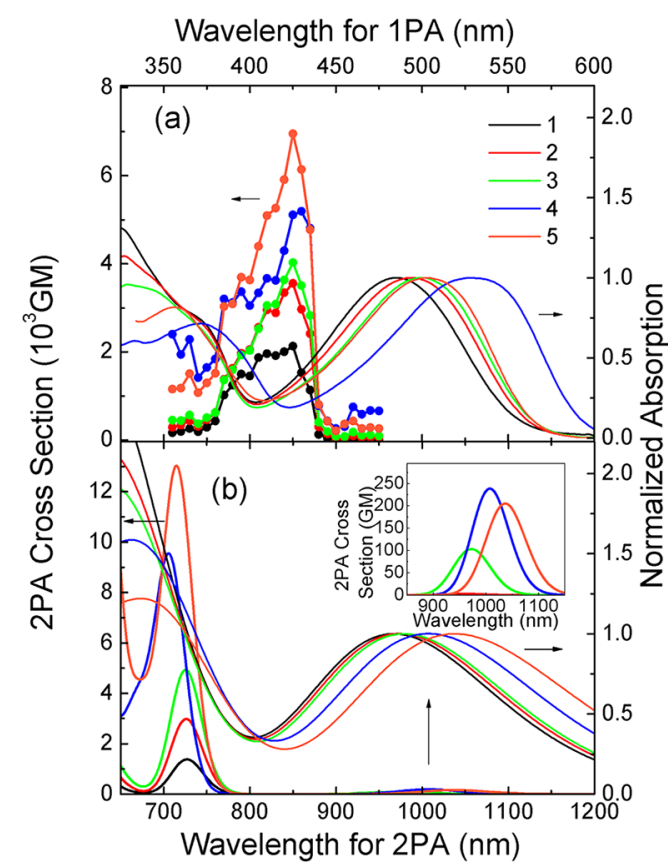

Figure 4. Two-photon excitation spectra of chromophores $\mathbf{1 - 5}$ in toluene (A) and the calculated two-photon excitation spectra (B) by using ZINDO methods. Inset of panel B shows the enlarged 2PA spectra from 850 to $1200 \mathrm{~nm}$. Normalized 1PA spectra are also shown for comparison.

calculated 2PA energy levels and 2PA cross-sections of chromophores 1-5. As mentioned above, the excited states in the 2PA spectra above $900 \mathrm{~nm}$ belong to the lowest ICT states. According to the eq 21, the expression of the majority part of the $2 \mathrm{PA}$ cross-section for the low-energy ICT states could be written as $\delta_{0 \rightarrow f} \propto\left|\left(\boldsymbol{\mu}_{f f}-\boldsymbol{\mu}_{00}\right) \cdot \boldsymbol{\mu}_{f 0} /\left(\omega_{f 0}-\omega\right)\right|^{2}=\mid \boldsymbol{\mu}_{f f}-$ $\left.\boldsymbol{\mu}_{00}\right|^{2}\left|\boldsymbol{\mu}_{f 0}\right|^{2} \cos ^{2} \alpha /\left(\omega_{f 0}-\omega\right)^{2}$, where $\boldsymbol{\mu}_{f 0}$ is the transition dipole moment from the ground state $(0)$ to the final state $(f), \boldsymbol{\mu}_{f f}$ is the dipole moment of the final state, $\alpha$ is the angle between $\left(\boldsymbol{\mu}_{f f}\right.$ $\left.-\boldsymbol{\mu}_{00}\right)$ and $\boldsymbol{\mu}_{f 0},\left(\omega_{f 0}-\omega\right)$ is the difference between the excitation energy of the $0 \rightarrow f$ transition and the absorbed photons. The dipole moments of these low-lying excited states caused by charge-transfer are the main contributions to the strength of the absorption. Therefore, in the absorption region from 900 to $1200 \mathrm{~nm}$, it has also been shown in literatures that the $2 \mathrm{PA}$ cross-section of an acceptor- $\pi$--donor system can be described even with a simple two-state model involving the ground (0) and the low-energy ICT state $(f)$ without a substantial loss of accuracy. ${ }^{71}$

For chromophore 1, expression for the main part of the 2PA cross-sections for the lowest ICT state in the region above 900 $\mathrm{nm}$ could be written as $\delta_{0 \rightarrow 1} \propto\left|\left(\boldsymbol{\mu}_{11}-\boldsymbol{\mu}_{00}\right) \cdot \boldsymbol{\mu}_{10} /\left(\omega_{10}-\omega\right)\right|^{2}$. With the quadruple structure of chromophore 1, the major donor groups of these low-energy ICT states are the triphenylamine cores, and the acceptor groups are all benzothiadiazole groups in the middle of the branch. The difference of dipole moments $\left(\boldsymbol{\mu}_{11}-\boldsymbol{\mu}_{00}\right)$ between the ground state and the excited state becomes small and even approaches zero in the case of high symmetry of the quadruple chromophore, leading to a small 2PA cross-section ( $\sim 1 \mathrm{GM})$. The calculated 2PA cross-sections of the chromophores 1-5 are shown in Figure 4 and Table S1 in Supporting Information.

For multibranched chromophores (chromophores 2-5), the main contribution terms of the $2 \mathrm{PA}$ cross-section of the low- 
energy ICT states in the region above $900 \mathrm{~nm}$ includes both $\left(\boldsymbol{\mu}_{11}-\boldsymbol{\mu}_{00}\right) \cdot \boldsymbol{\mu}_{10} /\left(\omega_{10}-\omega\right)$ and $\left(\boldsymbol{\mu}_{22}-\boldsymbol{\mu}_{00}\right) \cdot \boldsymbol{\mu}_{20} /\left(\omega_{20}-\omega\right)$. Because of the $C_{2}$ symmetry nature of chromophore 2 , these terms close to 0 , therefore, are similar to chromophore $\mathbf{1}$; chromophore 2 also has small 2PA cross-sections (for both chromophore $\mathbf{1}$ and 2, the calculated 2PA cross-section is less than 1GM) in the region above $900 \mathrm{~nm}$. However, for 3branched chromophores $3-5$, not only are $\left(\boldsymbol{\mu}_{11}-\boldsymbol{\mu}_{00}\right)$ and $\left(\boldsymbol{\mu}_{22}\right.$ $\left.-\boldsymbol{\mu}_{00}\right)$ much larger than $\left(\boldsymbol{\mu}_{11}-\boldsymbol{\mu}_{00}\right)$ of the chromophore $\mathbf{1}$, but also $\boldsymbol{\mu}_{10}$ and $\boldsymbol{\mu}_{20}$ of $C_{3 h}$ chromophores 3-5 are larger than $\boldsymbol{\mu}_{10}$ of chromophore 1. Therefore, the terms $\left(\boldsymbol{\mu}_{11}-\boldsymbol{\mu}_{00}\right) \cdot \boldsymbol{\mu}_{10} /\left(\omega_{10}-\right.$ $\omega)$ and $\left(\boldsymbol{\mu}_{22}-\boldsymbol{\mu}_{00}\right) \cdot \boldsymbol{\mu}_{20} /\left(\omega_{20}-\omega\right)$ of chromophores 3-5 contribute significantly to the 2PA cross-section of low-energy ICT states (45 GM for chromophore 3, $53 \mathrm{GM}$ for of chromophore 4, and 47 GM for chromophore 5, respectively).

Furthermore, for chromophores 3-5, the better charge transfer efficiency the bridge has, the larger $\left|\boldsymbol{\mu}_{f f}-\boldsymbol{\mu}_{00}\right|$ is; the dipole moments $\left(\boldsymbol{\mu}_{f f}-\boldsymbol{\mu}_{00}\right)$ and $\boldsymbol{\mu}_{f 0}$ are more collinear, and thus the larger absorption cross-section chromophore could be obtained. Therefore, in the region of 900-1200 nm, chromophore 4 has the largest 2PA cross-section (53 GM (calcd)). When the charge transfer efficiency of the bridge is not so effective, such as what occurs in chromophores $\mathbf{3}$ and $\mathbf{5}$, the determining factors $\left|\boldsymbol{\mu}_{f f}-\boldsymbol{\mu}_{00}\right|,\left|\boldsymbol{\mu}_{f 0}\right|$, and $\cos \alpha$ become similar. In addition, chromophore 5 has a larger $\left|\boldsymbol{\mu}_{f 0}\right|$ compared to that of chromophore 3 , so chromophore 5 has the second largest 2PA cross-section (47 GM (calcd)) in low-energy ICT states.

According to eq 21 , the main contribution to the 2PA crosssection in the region of the 770-870 nm band (corresponding to the high-energy ICT state region of 1PA from 385 to 435 $\mathrm{nm})$ is from the term $\boldsymbol{\mu}_{f m} \cdot \boldsymbol{\mu}_{m 0} /\left(\omega_{m 0}-\omega\right)$. In such dominating contributions, the final excited states $(f)$ are the $C_{3 h}$ symmetrical high-energy ICT states (shown in Table S2 in the Supporting Information). The large amplitude of $\boldsymbol{\mu}_{f m}$ and $\boldsymbol{\mu}_{m 0}$ is favorable for 2PA with a large cross-section, and when the two transitions $\boldsymbol{\mu}_{f m}$ and $\boldsymbol{\mu}_{m 0}$ are collinear, $\boldsymbol{\mu}_{f m} \cdot \boldsymbol{\mu}_{m 0}$ reaches its maximum. This particular symmetry of these states guarantees the colinearity of $\boldsymbol{\mu}_{f m}$ and $\boldsymbol{\mu}_{m 0}$ transitions. As the amplitudes of the transition dipole moments from the ground state to the lowest lying ICT excited states are very large, these transition dipole moments play the critical role (as $\boldsymbol{\mu}_{m 0}$ ) in dominating contribution to the large 2PA of the high-energy ICT state. For all the high-energy ICT states of chromophores 1 to $5,\left(\omega_{m 0}-\right.$ $\omega)$ within the term $\boldsymbol{\mu}_{f m} \cdot \boldsymbol{\mu}_{m 0} /\left(\omega_{m 0}-\omega\right)$ is almost the same; thus, our theoretical calculation results predict that, in chromophores $\mathbf{1}$ to $\mathbf{5}$, to a certain extent, $\left|\boldsymbol{\mu}_{m 0}\right|$ values could decide the 2PA cross-section of the 770-870 $\mathrm{nm}$ band.

As shown in Figure 4, the experimental 2PA spectra agrees well with our theoretical prediction for chromophores 1 to 5 , $2 \mathrm{PA}$ in the region of $770-870 \mathrm{~nm}$ are dominated by absorption. By comparing chromophores 1-3, we found, that the coupling of branches increases the transition dipole moment from the ground state to the lowest lying ICT states, where, for chromophore $\mathbf{1},\left|\boldsymbol{\mu}_{10}\right|$ is $4.08 \mathrm{D}$, and for chromophore 2, $\left|\boldsymbol{\mu}_{10}\right|$ and $\left|\boldsymbol{\mu}_{20}\right|$ are 5.25 and $2.49 \mathrm{D}$, respectively. For chromophore 3, both $\left|\boldsymbol{\mu}_{10}\right|$ and $\left|\boldsymbol{\mu}_{20}\right|$ are $5.00 \mathrm{D}$. As the 2PA cross-section in the region of $770-870 \mathrm{~nm}$ is mainly determined by $\boldsymbol{\mu}_{m 0}$, the calculated 2PA cross-section predicts a succesive increase of 2PA cross-sections from chromophores 1 to 3 as 982 GM, 2122 GM, and 2468 GM, correspondingly, while the observed 2PA cross-sections for chromophores 1, 2, and 3 are 2166 GM, 3591 GM, and 4064 GM, respectively. The observed 2PA cross-section ratios obtained for $\sigma_{2} / \sigma_{1}$ and $\sigma_{3} / \sigma_{1}$ are 1.66 and 1.88 , slightly lower than the theoretical predication (2.16 and 2.51). Furthermore, the influence of different $\pi$ bridge groups on the 2PA could be further investigated by comparing the computational and experimental data of chromophores 3-5. The idea that additional $\pi$ conjugated spacers promotethe $2 \mathrm{PA}$ cross-section is supported by both our experiment and computational data. It is found that the introduction of a double- and triple-bond linker in chromophores 4 and 5 leads to an increase of 2PA crosssections relative to the $2 \mathrm{PA}$ of chromophore 3 by a factor of 1.29 and 1.72 from experimental and 2.20 and 3.30 from calculation. The overestimated 2PA cross-section of 3-branched chromophores might be caused by the neglection of the impact of solvation on the molecular configurations. The plane conjugation configuration in vacuum (considered in calculation) might be broken in solution (experiment).

As shown in Figure 4, not only the absorption maximum at $850 \mathrm{~nm}(2.9 \mathrm{eV}$, corresponding to the high-energy ICT states) but also the shoulder peak at $780 \mathrm{~nm}(3.2 \mathrm{eV}$, corresponding to the low-energy states) are enhanced significantly for chromophores $\mathbf{4}$ and $\mathbf{5}$ as compared with chromophore 3 . This indicates that, when double- and triple-bond linkers are used between the donor and acceptor, a more pronounced charge transfer could be obtained, and the transition dipole moment would be enlarged, leading to the enhanced 2PA. The enhanced coupling within chromophores 4 or 5 relative to chromophore $\mathbf{3}$ is also evidenced from the fluorescence lifetimes (see Table 1), where the lifetimes are about $3.4 \mathrm{~ns}$ for chromophore 4, $5.2 \mathrm{~ns}$ for chromophore 5, and $6.6 \mathrm{~ns}$ for chromophore 3, respectively. The shortened fluorescence lifetime indicates the stronger coupling between conjugated branches within chromophores $\mathbf{4}$ and $\mathbf{5}$ compared to that in chromophore 3 .

\section{CONCLUSIONS}

In a series of branched quadrupolar ICT molecules (chromophores 1-5), there are two typical ICT states, the low-energy ICT states and high-energy ICT states. For the high-energy ICT states, the excitation is mostly attributed to the charge transfer from triphenylamine moieties on the end of branches to the benzothiadiazole moieties; whereas for the lowenergy ICT states, the excitation was mostly attributed to the charge transfer from the triphenylamine core to the benzothiadiazole moieties.

The one-photon UV-vis spectra and fluorescence spectra are mainly determined by low-energy ICT states. Shifting or splitting of the spectral band represents the strength of interbranched interaction. Except for chromophore 4, no remarkable shifting or splitting in the excited states was observed in normalized steady-state absorption and fluorescence spectra and solvatochromism measurement. The similar fluorescence and solvation behaviors between monomer and branched chromophores (dimer and trimer) imply that the interaction among branches is not strong enough to make a significant distinction between these molecules because of the weak interaction and intrinsic structural disorder in branched molecules.

The directions of transition dipole moments of both lowenergy ICT states and high-energy ICT states could be predicted by the Frenkel exciton model, which is confirmed by comparing the results from TD-DFT calculation with anisotropy fluorescence excitation spectra. When high-energy 
ICT states are excited, electron-hole pair is generated at triphenylamine moieties at the end of branches, the hole then transfers to the triphenylamine core before emission because, once the energy transfer from high-energy to low-energy ICT states occurred, the anisotropy values of the high-energy ICT states become relatively lower than that of the low-energy ICT states.

Furthermore, for the complementary measurement of the 1PA property, 2PA measurements were made to determine the detailed spectral properties of the high-energy ICT states, where the IPA of the high-energy ICT states is very weak and hidden from the strong 1PA of the low-energy ICT state. Although both low- and high-energy ICT states are 2PA active, it is found that $2 \mathrm{PA}$ cross-sections of high-energy ICT states are much larger than those of low-energy ICT states, where the increased transition dipole moment plays a critical role in the observed 2PA cross-sections for both low-energy and highenergy ICT states in the multibranched quadrupolar chromophores.

\section{ASSOCIATED CONTENT}

\section{S Supporting Information}

Computational results about the energy levels of one-photon and two-photon absorptions, calculated fluorescence excitation anisotropy, and synthesis of the chromophores 1-5. This material is available free of charge via the Internet at http:// pubs.acs.org.

\section{AUTHOR INFORMATION}

\section{Corresponding Author}

*Email: andong@iccas.ac.cn (A.X.); guoqj@iccas.ac.cn (Q.G.); sheng@mail.nctu.edu.tw (S.L.).

\section{Notes}

The authors declare no competing financial interest.

\section{ACKNOWLEDGMENTS}

This work was financially supported by NSFC, 973 Programs, and Chinese Academy of Sciences. D.A. and S.V. thank CAS for support from the CAS Research Fellowship for International Young Researchers.

\section{REFERENCES}

(1) Lai, R. Y.; Fabrizio, E. F.; Lu, L. D.; Jenekhe, S. A.; Bard, A. J. J. Am. Chem. Soc. 2001, 123, 9112.

(2) Sun, X. B.; Liu, Y. Q.; Xu, X. J.; Yang, C. H.; Yu, G.; Chen, S. Y.; Zhao, Z. H.; Qiu, W. F.; Li, Y. F.; Zhu, D. B. J. Phys. Chem. B 2005, 109, 10786.

(3) Tang, C. W.; Vanslyke, S. A.; Chen, C. H. J. Appl. Phys. 1989, 65, 3610 .

(4) Oregan, B.; Gratzel, M. Nature 1991, 353, 737.

(5) Loo, Y. L. AIChE J. 2007, 53, 1066.

(6) He, C.; He, Q. G.; He, Y. J.; Li, Y. F.; Bai, F. L.; Yang, C. H.; Ding, Y. Q.; Wang, L. X.; Ye, J. P. Sol. Energy Mater. Sol. Cells 2006, 90, 1815.

(7) Chung, S. J.; Rumi, M.; Alain, V.; Barlow, S.; Perry, J. W.; Marder, S. R. J. Am. Chem. Soc. 2005, 127, 10844.

(8) Bartholomew, G. P.; Rumi, M.; Pond, S. J. K.; Perry, J. W.; Tretiak, S.; Bazan, G. C. J. Am. Chem. Soc. 2004, 126, 11529.

(9) Chung, S. J.; Lin, T. C.; Kim, K. S.; He, G. S.; Swiatkiewicz, J.; Prasad, P. N.; Baker, G. A.; Bright, F. V. Chem. Mater. 2001, 13, 4071.

(10) Ma, X. N.; Yan, L. Y.; Wang, X. F.; Guo, Q. J.; Xia, A. D. Phys. Chem. Chem. Phys. 2011, 13, 17273.

(11) Yi, Y. P.; Li, Q. X.; Zhu, L. Y.; Shuai, Z. G. J. Phys. Chem. A 2007, 111, 9291.
(12) Jia, M.; Ma, X.; Yan, L.; Wang, H.; Guo, Q.; Wang, X.; Wang, Y.; Zhan, X.; Xia, A. J. Phys. Chem. A 2010, 114, 7345.

(13) Padilha, L. A.; Webster, S.; Przhonska, O. V.; Hu, H.; Peceli, D.; Rosch, J. L.; Bondar, M. V.; Gerasov, A. O.; Kovtun, Y. P.; Shandura, M. P.; Kachkovski, A. D.; Hagan, D. J.; Van Stryland, E. W. J. Mater. Chem. 2009, 19, 7503.

(14) Rubio-Pons, O.; Luo, Y.; Agren, H. J. Chem. Phys. 2006, 124.

(15) Goodson, T. G. Acc. Chem. Res. 2004, 38, 99.

(16) Kim, H. M.; Cho, B. R. Chem. Commun. 2009, 153.

(17) He, G. S.; Tan, L. S.; Zheng, Q.; Prasad, P. N. Chem. Rev. 2008, $108,1245$.

(18) Bhaskar, A.; Ramakrishna, G.; Lu, Z. K.; Twieg, R.; Hales, J. M.; Hagan, D. J.; Van Stryland, E.; Goodson, T. J. Am. Chem. Soc. 2006, 128,11840 .

(19) Chung, S. J.; Kim, K. S.; Lin, T. H.; He, G. S.; Swiatkiewicz, J.; Prasad, P. N. J. Phys. Chem. B 1999, 103, 10741.

(20) Macak, P.; Luo, Y.; Norman, P.; Agren, H. J. Chem. Phys. 2000, $113,7055$.

(21) Chen, L. X.; Jager, W. J. H.; Gosztola, D. J.; Niemczyk, M. P.; Wasielewski, M. R. J. Phys. Chem. B 2000, 104, 1950.

(22) Panthi, K.; Adhikari, R. M.; Kinstle, T. H. J. Phys. Chem. A 2010, $114,4542$.

(23) Chen, L. X.; Jager, W. J. H.; Niemczyk, M. P.; Wasielewski, M. R. J. Phys. Chem. A 1999, 103, 4341.

(24) Hagberg, D. P.; Marinado, T.; Karlsson, K. M.; Nonomura, K.; Qin, P.; Boschloo, G.; Brinck, T.; Hagfeldt, A.; Sun, L. J. Org. Chem. 2007, 72, 9550.

(25) Katan, C.; Tretiak, S.; Werts, M. H. V.; Bain, A. J.; Marsh, R. J.; Leonczek, N.; Nicolaou, N.; Badaeva, E.; Mongin, O.; BlanchardDesce, M. J. Phys. Chem. B 2007, 111, 9468.

(26) Katan, C.; Terenziani, F.; Mongin, O.; Werts, M. H. V.; Porrès, L.; Pons, T.; Mertz, J.; Tretiak, S.; Blanchard-Desce, M. J. Phys. Chem. A 2005, 109, 3024.

(27) Terenziani, F.; Katan, C.; Badaeva, E.; Tretiak, S.; BlanchardDesce, M. Adv. Mater. 2008, 20, 4641.

(28) Aumiler, D.; Wang, S.; Chen, X.; Xia, A. J. Am. Chem. Soc. 2009, $131,5742$.

(29) Loh, Z.-H.; Miller, S. E.; Chang, C. J.; Carpenter, S. D.; Nocera, D. G. J. Phys. Chem. A 2002, 106, 11700.

(30) Song, N. W.; Cho, H. S.; Yoon, M. C.; Aratani, N.; Osuka, A.; Kim, D. Bull. Korean Chem. Soc. 2002, 23, 271.

(31) Yang, Y.; Zhou, Y.; He, Q. G.; He, C.; Yang, C. H.; Bai, F. L.; Li, Y. F. J. Phys. Chem. B 2009, 113, 7745.

(32) He, C.; He, Q. G.; Yi, Y. P.; Wu, G. L.; Bai, F. L.; Shuai, Z. G.; Li, Y. F. J. Mater. Chem. 2008, 18, 4085.

(33) Wan, Y.; Yan, L.; Zhao, Z.; Ma, X.; Guo, Q.; Jia, M.; Lu, P.; Ramos-Ortiz, G.; Maldonado, J. L.; Rodríguez, M.; Xia, A. J. Phys. Chem. B 2010, 114, 11737.

(34) Lakowicz, J. R.; Masters, B. R. J. Biomed. Opt. 2008, 13, 029901.

(35) Xu, C.; Webb, W. W. J. Opt. Soc. Am. B 1996, 13, 481.

(36) Albota, M. A.; Xu, C.; Webb, W. W. Appl. Opt. 1998, 37, 7352.

(37) Rumi, M.; Ehrlich, J. E.; Heikal, A. A.; Perry, J. W.; Barlow, S.; Hu, Z.; McCord-Maughon, D.; Parker, T. C.; Röckel, H.; Thayumanavan, S.; Marder, S. R.; Beljonne, D.; Brédas, J.-L. J. Am. Chem. Soc. 2000, 122, 9500.

(38) Davydov, A. S. Soviet Phys. Usp. 1964, 7, 145.

(39) Beljonne, D.; Wenseleers, W.; Zojer, E.; Shuai, Z. G.; Vogel, H.; Pond, S. J. K.; Perry, J. W.; Marder, S. R.; Bredas, J. L. Adv. Funct. Mater. 2002, 12, 631.

(40) Silinšs, E.; Čápek, V. Organic Molecular Crystals: Interaction, Localization, and Transport Phenomena; American Institute of Physics: New York, 1994.

(41) Davydov, A. S. Theory of Molecular Excitons; Plenum Press: New York, 1971.

(42) Terenziani, F.; Sissa, C.; Painelli, A. J. Phys. Chem. B 2008, 112, 5079.

(43) Terenziani, F.; Morone, M.; Gmouh, S.; Blanchard-Desce, M. ChemPhysChem 2006, 7, 685. 
(44) Terenziani, F.; Le Droumaguet, C.; Katan, C.; Mongin, O.; Blanchard-Desce, M. ChemPhysChem 2007, 8, 723.

(45) Pettit, D. L.; Wang, S. S. H.; Gee, K. R; Augustine, G. J. Neuron 1997, 19, 465.

(46) Lin, S. H.; Fujimura, Y.; Neusser, H. J.; Schlag, E. W. Multiphoton Spectroscopy of Molecules; Academic Press: New York, 1984.

(47) Jagatap, B. N.; Meath, W. J. J. Opt. Soc. Am. B 2002, 19, 2673.

(48) Dick, B.; Hohlneicher, G. J. Chem. Phys. 1982, 76, 5755.

(49) Thirunamachandran, D. P. C. a. T. Molecular Quantum

Electrodynamics: An Introduction to Radiation-Molecule Interactions;

Dover Publications: London, U.K., 1984.

(50) Sun, M. T. J. Chem. Phys. 2006, 124.

(51) Persson, N. K.; Sun, M. T.; Kjellberg, P.; Pullerits, T.; Inganas, O. J. Chem. Phys. 2005, 123.

(52) Wang, X. F.; Zhang, X. R.; Wu, Y. S.; Zhang, H. P.; Ai, X. C.; Wang, Y.; Sun, M. T. Chem. Phys. Lett. 2007, 436, 280.

(53) Frisch, M. J.; Trucks, G. W.; Schlegel, H. B.; Scuseria, G. E.; Robb, M. A.; Cheeseman, J. R.; Montgomery, J. A., Jr.; Vreven, T.; Kudin, K. N.; Burant, J. C.; Millam, J. M.; Iyengar, S. S.; Tomasi, J.; Barone, V.; Mennucci, B.; Cossi, M.; Scalmani, G.; Rega, N.; Petersson, G. A.; Nakatsuji, H.; Hada, M.; Ehara, M.; Toyota, K.; Fukuda, R.; Hasegawa, J.; Ishida, M.; Nakajima, T.; Honda, Y.; Kitao, O.; Nakai, H.; Klene, M.; Li, X.; Knox, J. E.; Hratchian, H. P.; Cross, J. B.; Bakken, V.; Adamo, C.; Jaramillo, J.; Gomperts, R.; Stratmann, R. E.; Yazyev, O.; Austin, A. J.; Cammi, R.; Pomelli, C.; Ochterski, J. W.; Ayala, P. Y.; Morokuma, K.; Voth, G. A.; Salvador, P.; Dannenberg, J. J.; Zakrzewski, V. G.; Dapprich, S.; Daniels, A. D.; Strain, M. C.; Farkas, O.; Malick, D. K.; Rabuck, A. D.; Raghavachari, K.; Foresman, J. B.; Ortiz, J. V.; Cui, Q.; Baboul, A. G.; Clifford, S.; Cioslowski, J.; Stefanov, B. B.; Liu, G.; Liashenko, A.; Piskorz, P.; Komaromi, I.; Martin, R. L.; Fox, D. J.; Keith, T.; Al-Laham, M. A.; Peng, C. Y.; Nanayakkara, A.; Challacombe, M.; Gill, P. M. W.; Johnson, B.; Chen, W.; Wong, M. W.; Gonzalez, C.; Pople, J. A. Gaussian 03, Revision E.01; Gaussian, Inc.: Wallingford, CT, 2004.

(54) Ranasinghe, M. I.; Varnavski, O. P.; Pawlas, J.; Hauck, S. I.; Louie, J.; Hartwig, J. F.; Goodson, T. J. Am. Chem. Soc. 2002, 124, 6520.

(55) Gong, Y.; Guo, X.; Wang, S.; Su, H.; Xia, A.; He, Q.; Bai, F. J. Phys. Chem. A 2007, 111, 5806.

(56) Kato, S.; Matsumoto, T.; Shigeiwa, M.; Gorohmaru, H.; Maeda, S.; Ishi-i, T.; Mataka, S. Chem.-Eur. J. 2006, 12, 2303.

(57) Moonen, N. N. P.; Gist, R.; Boudon, C.; Gisselbrecht, J. P.; Seiler, P.; Kawai, T.; Kishioka, A.; Gross, M.; Irie, M.; Diederich, F. Org. Biomol. Chem. 2003, 1, 2032.

(58) Li, N.; Jia, K.; Wang, S.; Xia, A. J. Phys. Chem. A 2007, 111, 9393.

(59) Varnavski, O. P.; Ostrowski, J. C.; Sukhomlinova, L.; Twieg, R. J.; Bazan, G. C.; Goodson, T. J. Am. Chem. Soc. 2002, 124, 1736.

(60) Kumar, S.; Rao, V. C.; Rastogi, R. C. Spectrochim. Acta, Part A 2001, 57, 41 .

(61) Lee, W.-H.; Lee, H.; Kim, J.-A.; Choi, J.-H.; Cho, M.; Jeon, S.-J.; Cho, B. R. J. Am. Chem. Soc. 2001, 123, 10658.

(62) Huang, Y.; Cheng, T.; Li, F.; Luo, C.; Huang, C.-H.; Cai, Z.; Zeng, X.; Zhou, J. J. Phys. Chem. B 2002, 106, 10031.

(63) Drobizhev, M.; Meng, F. Q.; Rebane, A.; Stepanenko, Y.; Nickel, E.; Spangler, C. W. J. Phys. Chem. B 2006, 110, 9802.

(64) Katan, C.; Charlot, M.; Mongin, O.; Le Droumaguet, C. L.; Jouikov, V.; Terenziani, F.; Badaeva, E.; Tretiak, S.; Blanchard-Desce, M. J. Phys. Chem. B 2010, 114, 3152.

(65) Terenziani, F.; Mongin, O.; Katan, C.; Bhatthula, B. K. G.; Blanchard-Desce, M. Chem.-Eur. J. 2006, 12, 3089.

(66) Sissa, C.; Painelli, A.; Blanchard-Desce, M.; Terenziani, F. J. Phys. Chem. B 2011, 115, 7009.

(67) Prazeres, T. J. V.; Fedorov, A.; Barbosa, S. P.; Martinho, J. M. G.; Berberan-Santos, M. N. J. Phys. Chem. A 2008, 112, 5034.

(68) Guo, X.; Wang, S.; Xia, A.; Su, H. J. Phys. Chem. A 2007, 111, 5800.
(69) Verbouwe, W.; Van der Auweraer, M.; De Schryver, F. C.; Piet, J. J.; Warman, J. M. J. Am. Chem. Soc. 1998, 120, 1319.

(70) Latterini, L.; De Belder, G.; Schweitzer, G.; Van der Auweraer, M.; De Schryver, F. C. Chem. Phys. Lett. 1998, 295, 11.

(71) Albota, M.; Beljonne, D.; Bredas, J. L.; Ehrlich, J. E.; Fu, J. Y.; Heikal, A. A.; Hess, S. E.; Kogej, T.; Levin, M. D.; Marder, S. R.; McCord-Maughon, D.; Perry, J. W.; Rockel, H.; Rumi, M.; Subramaniam, C.; Webb, W. W.; Wu, X. L.; Xu, C. Science 1998, 281, 1653.

(72) Lin, C. K.; Wang, Y. H.; Chang, H. C.; Hayashi, M.; Lin, S. H. J. Chem. Phys. 2008, 129.

(73) Yi, Y. P.; Zhu, L. Y.; Shuai, Z. G. Macromol. Theory Simul. 2008, $17,12$.

(74) Zhu, L. Y.; Yi, Y. P.; Shuai, Z. G.; Schmidt, K.; Zojer, E. J. Phys. Chem. A 2007, 111, 8509.

(75) Zhou, X.; Ren, A. M.; Feng, J. K.; Shuai, Z. G. J. Photochem. Photobiol., A 2005, 172, 126.

(76) Zhu, L. Y.; Yi, Y. P.; Shuai, Z. G.; Bredas, J. L.; Beljonne, D.; Zojer, E. J. Chem. Phys. 2006, 125.

(77) Shuai, Z. G.; Beljonne, D.; Bredas, J. L. J. Chem. Phys. 1992, 97, 1132.

(78) He, Y. H.; Hui, R. J.; Yi, Y. P.; Shuai, Z. G. Acta Phys.-Chim. Sin. 2008, 24, 565. 